\title{
LA PORTÉE RHÉTORIQUE ET LITTÉRAIRE DES PROLALIAI CHEZ LUCIEN, DANS HERODOTUS, SCYTHA ET HARMONIDES
}

\author{
Jean-Luc Vix \\ Université de Strasbourg
}

RESUMO: $\mathrm{O}$ artigo se propõe a explorar os procedimentos adotados por Luciano em certos discursos breves, as prolaliaí, destinadas a captar a benevolência do público. Parece que, se o rétor de Samósata emprega de bom grado tópoi nestes textos, ele não é, contudo, tributário das regras estabelecidas que se aprendem nas escolas de retórica. A investigação tenta evidenciar paralelos, na composição e no objetivo dos discursos, bem como nos exercícios escolares, os progymnásmata, que lhes são aplicados. A escrita de Luciano parece, assim, claramente ligada aos preceitos dos teóricos da retórica, mas, além das aparências formais, também se ocultam dissonâncias entre as diferentes prolaliaí, o que as particulariza. É à descoberta dessas tonalidades próprias a cada obra que se vincula o artigo em uma segunda parte, intentando descobrir a originalidade de cada gesto de escrita. São, ainda, metas descobrir sua particularidade, sua tonalidade própria, bem como a singularidade de Luciano no seio da Segunda Sofística.

PALAVRAS-CHAVE: prolaliaí; retórica; Segunda Sofística; exercícios preparatórios (= progymnásmata); paideía.

ême si les études concernant les prolaliai de Lucien ne sont pas très nombreuses, ${ }^{1}$ leur existence même tout au long du $\mathrm{XX}^{\mathrm{e}}$ siècle, prouve, s'il en était besoin, que ces discours n'ont cessé d'intriguer la communauté scientifique, dans la mesure où leur groupe constitue une particularité, dans ce qui nous reste de la littérature de cette époque.

^jean-luc.vix@wanadoo.fr

${ }^{1}$ Voir la bibliographie à la dernière page. 
Karl Mras, Die prolaliá bei den griechischen Schriftstellern, Wiener Studien LXIV, 1949, a tenté de comparer les prolaliai de Lucien avec d'autres écrits de la Seconde Sophistique qui pourraient aussi mériter ce titre, que ce soit de Dion de Pruse, ${ }^{2}$ de Libanios, de Thémistios, d'Himérios, ou encore de Chorikios, pour aboutir à la conclusion (p. 81) que les discours de Lucien sont uniques dans le monde grec, et ne peuvent être rapprochés parfois que des Florida d'Apulée, uniques dans le monde latin. Leur contenu les rendrait, selon Mras, peu susceptibles de comparaisons avec d'autres écrits de l'époque impériale.

\section{La prolalia: problème du genre et de la réception}

Définir la prolalia ${ }^{3}$ n'est pas chose aisée, les théoriciens n'utilisant jamais ce terme mais celui de lalia, de sorte qu'une confusion s'opère entre les deux notions. ${ }^{4}$ Il semble assuré que lalia et prolalia sont des créations de la Seconde Sophistique. ${ }^{5}$ Le mot, à l'époque impériale, semble essentiellement appartenir au domaine du genre épidictique, comme on le constate à travers le traité de Ménandros II,

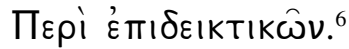

Ménandros II aborde la lalia sous deux aspects, 388-394 Peri lalias et 395-399 Peri propemptikês, discours de départ. Le théoricien n'utilise jamais le terme de prolalia, mais on se rend compte qu'il opère une distinction entre deux sortes de laliai: celles qui constituent des discours autonomes, et celles qui appellent un second discours (393, 24-26; 434,

\footnotetext{
${ }^{2}$ Cf. Stock, op. cit., p. 41-66; Mras, op. cit., p. 74-77. Pour d'autres sophistes apparaissant chez Philostrate, cf. ibid., p. 85-94.

${ }^{3}$ La seule étude d'ensemble sur les Prolaliai est la dissertation de Stock, op. cit.. La liste des prolaliai qu'il donne (p. 11-105) est perçue comme trop étendue à l'heure actuelle.

${ }^{4}$ Pour une étude sur la lalia et la prolalia voir Pernot, op. cit., 1993, (t. II, p. 546 ss.).

${ }^{5}$ Même si le terme de lalia apparaît pour la première fois chez Aristophane, Nuées 931, dans sa critique contre les sophistes.

${ }^{6}$ Cf. Russell; Wilson, op. cit.. Sur l'appartenance de la lalia au genre épidictique, voir Bompaire, op. cit., p. 286-287.

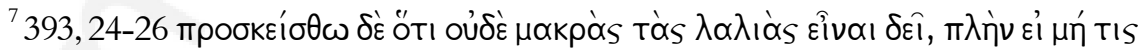

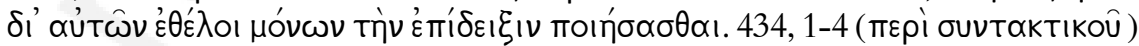

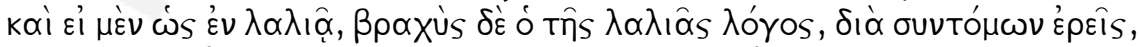

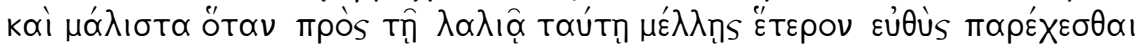

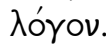


$1-9)^{7}$ et sont alors une sorte de prologue, une prolalia. Mais dans les deux cas, ces discours se caractérisent par leur brièveté (oủ Mokpós 393, 25). Dans ses Vies des sophistes, Philostrate mentionne fréquemment des cours ou épideixeis qui comportent "deux parties: un petit discours d'introduction (dialexis, dialegesthai), puis la déclamation proprement dite (meletê, meletan, agônizesthai). "Dialexis est donc le terme technique désignant la prolalie"8 pratiquée par nombre de sophistes.

Nous ne disposons d'aucune attestation permettant de valider le terme "prolalia" avant les $\mathrm{III}^{\mathrm{e}}-\mathrm{IV}^{\mathrm{e}}$ s. L'apparition la plus ancienne du mot vient de la Souda (T 550, éd. Adler) qui signale que Tiberios (III$\mathrm{IV}^{\mathrm{e}}$ s.) fut l'auteur d'un traité sur les prolalia et les prooimia. ${ }^{9}$ Dans ce témoignage important, prolalia et prooimion figurent donc sur le même plan, comme deux appellations d'une même réalité. Cette mention ne résout pourtant pas toutes les questions. Mais si l'on accepte que le

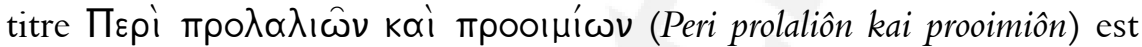
dû au rhéteur du III-IV ${ }^{\mathrm{e}}$. ap. J. C., ${ }^{10}$ le mot, par conséquent, aurait été consacré un ou deux siècles après Lucien. Par ailleurs, ce titre a été donné à deux courts textes de Lucien, Bacchus et Hercules, par les manuscrits parmi les plus anciens (Vaticanus graecus 90, $\mathrm{X}^{\mathrm{e}}$ s.; Marcianus graecus 434, $\mathrm{X}_{\mathrm{XI}}^{\mathrm{e}}$ s.; Mutininensis á. $\mathrm{V}$. 8.15, $\mathrm{XI}^{\mathrm{e}}$ s.; Vindobonensis phil. gr. 123, $\mathrm{XI}^{\mathrm{e}}$ s.). ${ }^{11}$ Mais si les attestations lexicales sont rares, la réalité des prolaliai l'est beaucoup moins au tournant de l'ère chrétienne.

La prolalia devient en effet un discours bref de caractère autonome, ${ }^{12}$ destiné à introduire une lecture ou récitation d'un discours plus important. Le lien avec les prooimia est, de par la fonction même

${ }^{8}$ Cf. Pernot, op. cit., 1993, (t. II, p. 553).

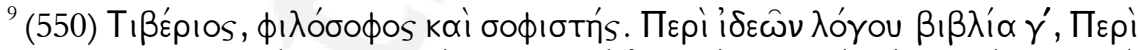

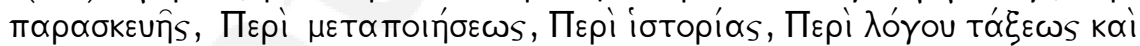

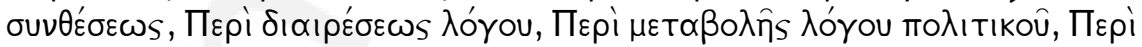

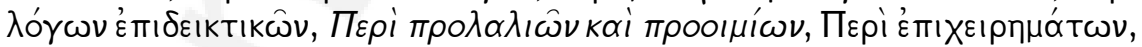

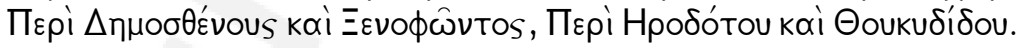

${ }^{10}$ Cf. Desbordes, op. cit., p. 276./ Spengel, op. cit., 1853-1856 (repr. Francfort-sur-le Main, 1966, p. 57-82)/Walz, op. cit., 1832-1836 (repr. Osnabrück, 1968, vol. VIII, p. 520-577).

${ }^{11}$ Cf. Nesselrath, op. cit., p. 110-140, p. 110, qui se réfere à l'ouvrage ancien de Stock, op. cit., et éd. CUF, t. 1: le sous-titre de prolalia apparaît dans ces manuscrits pour Bacchus et Hercules.

${ }^{12}$ Dans le Scythe $\int 9$ le télos du discours est clairement évoqué, signifiant ainsi qu'il se suffit à lui-même. 
des deux textes - des sortes d'exorde -, clairement visible: "Les séances oratoires commençaient d'ordinaire par une sorte d'introduction, de ton

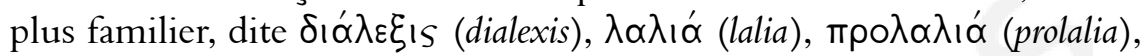
où les sophistes se présentaient au public en essayant de gagner sa bienveillance. Volontiers, ils introduisaient dans cet avant-propos un

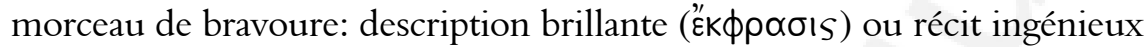
$(\delta ı \eta ́ \gamma \eta \mu \alpha) "{ }^{13}$ La prolalia serait, dans sa fonction de prologue - ou devrait être -, étroitement liée au discours principal, au moins par le sujet.

Sur l'origine des prolaliai, comme discours d'introduction indépendants, il faut sans doute prendre en compte l'habitude de rédiger des prooimia, des parties introductives, qui, peu ou prou, pouvaient parfois jouer un rôle similaire, en ce qui concerne la difficulté de tenir le discours par ex. (= thème de l'adunaton), mais sans pousser aussi loin que les prolaliai les parallèles avec des exemples illustres et sans acquérir l'autonomie qui sera une caractéristique des prolaliai. On peut, à titre d'exemple, citer le prologue du discours 33 d'Aristide, qui, sans être totalement autonome, pourrait être détaché du discours qui suit, et se situe donc entre prolalia et exorde. ${ }^{14}$ Du même auteur, l'exorde de l'Hymne à Sarapis a parfois été perçu comme une prolalia, tant le rapport avec le sujet principal est lâche et distendu. Le genre épidictique est donc marqué par cette spécificité d'un exorde, sans rapport obligatoire avec le sujet, ce qui permet de comprendre l'émergence de la prolalia comme genre autonome à l'époque impériale.

\section{Les prolaliai lucianesques}

A l'intérieur de la production de la Seconde Sophistique, les prolaliai de Lucien sont œuvres originales, ainsi que le souligne H. G. Nesselrath, ${ }^{15}$ qui évoque aussi bien leur structure que leur

${ }^{13}$ Cf. Boulanger, op. cit., p. 51.

${ }^{14}$ Cf. Stock, op. cit., p. 85, n ${ }^{\circ}$ 59. Le discours 33 d'Aelius Aristide est paru récemment dans une traduction française et un commentaire, Vix, 2010. Dans une problématique parallèle il convient également d'avoir à l'esprit le développement, depuis les âges classiques, de "l'exorde orné ou hors sujet", cf. Pernot, op. cit., 1993, p. 557; Aristote, Rhétorique III, 1414 a ss., passage dans lequel le Stagirite aborde le problème du prologue, élément nécessaire de tout discours: les réflexions d'Aristote soulignent, que, si l'attache entre l'exorde et le discours qui suit, n'est pas obligatoirement thématique, il est cependant nécessaire de lier les deux parties, par une transition.

${ }^{15}$ Cf. Nesselrath, op. cit., p. 111-140, p. 113. 
contenu, ou encore les innombrables références personnelles comme des marques spécifiques du Samosate.

\section{Définition d'un corpus}

Comme cela a été souligné plus haut, la délimitation elle-même des discours lucianesques susceptibles d'être analysés comme de prolaliai est délicate et a donné lieu à différentes suppositions, sur lesquelles il n'y a pas lieu de revenir ici. Dionysos et Hercules ont bien entendu un statut à part, vu le titre qui leur est donné par les manuscrits les plus anciens, ainsi que nous l'avons évoqué plus haut.

On s'accorde au minimum sur huit discours qui seraient indiscutablement des prolaliai: Bacchus (Stock, $\mathrm{n}^{\circ} 1$ ), Hercules (Stock $\mathrm{n}^{\circ} 2$ ), De electro sive de cycnis (Stock n $\left.{ }^{\circ} 3\right),{ }^{16}$ De dipsadibus (Stock no 4 ), Herodotus sive Aetion (Stock n ${ }^{\circ}$ 5, p 21), Zeuxis sive Antiochus (Stock n ${ }^{\circ}$ 6), Scytha sive hospes, Harmonides. ${ }^{17}$ D'autres discours font moins l'unanimité. C'est le cas de Somnium sive vita Luciani ${ }^{18}$ De domo, Prometheus es in verbis. ${ }^{19}$

De façon générale on pourrait dire que Lucien annonce fréquemment dans ces pièces, mais de manière assez relâchée, des

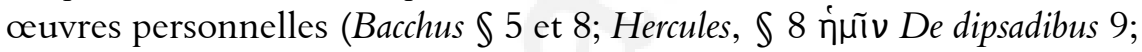
Herodotus $\ 7$; Scytha $\ 10$; Harmonides $\ 3$ ).

${ }^{16}$ Stock, op. cit., p. 20 remarque que la dernière phrase du De electro comporte le verbe prolégô $\left(\pi \rho \circ \lambda \varepsilon^{\prime} \gamma \omega\right)$, ce qui, selon lui, permet indubitablement de classer ce discours comme prologue. A sa suite, et en élargissant le propos, Pernot, op. cit., 1993, p. 550, souligne que le caractère d'introduction à une "épideixis" y est patent dans chacun des huit cas, notamment dans les phrases finales.

${ }^{17}$ Listes que l'on retrouve chez Rothstein, op. cit., p. 116, Mras, op. cit., p. 71, Pernot, op. cit., 1993, p. 550. Stock, op. cit., exclut Scytha sive hospes et Harmonides, p. 28-33.

${ }^{18}$ Discours considéré comme prolalia par Stock, op. cit., p. 25-28; réserves de Rothstein, op. cit., p. 117 et Bompaire, op. cit., p. 288, n. 5; Anderson, op. cit., p. 313315 , p. 314, n. 5, émet l'hypothèse qu'il pourrait s'agir d'un discours d'école. Considéré comme trop long par Branham, op. cit., p. 237-243, p. 238, n. 4. Il n'annonce pas d'autre discours, selon Pernot, op. cit., 1993, p. 555.

${ }^{19}$ Considérés comme prolaliai par Rothstein, op. cit., p. 117 et J. Bompaire, op. cit., p. 288, n. 5 (plus nuancé dans l'édition de la CUF, t. I, p. 149: Ce n'est pas une simple "prolalia" introductive). Stock, op. cit., considère que De domo n'est pas une prolalia, p. 34 de même que G. Anderson, op. cit., p. 314, n. 5, ainsi que R. B. Branham, op. cit., p. 238, n. 4. Ces deux derniers auteurs considèrent cependant que Prometheus es in verbis est une prolalia. 


\section{Enquête sur trois prolaliai: justification d'un corpus d'étude}

Le travail qui suit s'attachera à trois prolaliai, Herodotus, Scytha, et Harmonides.

À la suite de la remarque de Branham, ${ }^{20}$ on ne peut que remarquer que les études antérieures sur les prolaliai lucianesques ont ignoré la portée rhétorique et littéraire de ces œuvres. Or, un tel objectif semble plus réalisable à travers un corpus réduit, car prétendre englober dans une étude rhétorique l'ensemble des prolaliai serait illusoire et aurait pour seul effet de diluer les remarques. Réduire le champ d'investigation à quelques prolalies devrait donc permettre une observation plus fine. Restait donc à opérer une sélection.

Si l'on suit la classification proposée par J. Bompaire, on constate que, parmi les huit prolaliai reconnues comme telles, on peut repérer deux groupes: "Quatre de ces laliai sont غmıßarńpıo, sorte de prise de contact avec un public et un ${ }^{\alpha} \rho \chi \omega \nu$ (notable) étrangers, macédoniens dans Herodotus et Scytha, non précisé dans Harmonides et dans De Electro; au contraire dans le Zeuxis, les Dipsades, Dionysos et Hercules Lucien s'adresse à son auditoire habituel". Herodotus et Scytha se détachent au sein d'un groupe de quatre discours prononcés dans un environnement "étranger", puisque les deux prolaliai sont prononcés devant un public macédonien. Non seulement les deux discours sont proches géographiquement, mais il se pourrait qu'ils le soient également chronologiquement. Selon J. Schwartz ${ }^{21}$ les deux prolaliai dateraient de la même période, les années 166-168, et seraient par conséquent des œuvres de jeunesse du sophiste. La situation chronologique de Harmonides est peut-être plus difficile à cerner, mais est perçue également comme appartenant à la première partie de la carrière du Samosate par C. P. Jones, p. 169 et H. G. Nesselrath, p. 117 ("Herodotus sive Aetioni which must probably be taken closely together with Harmonides and Scytha"). On a vu, par ailleurs, que Harmonides appartient au groupe des quatre prolaliai tenues devant un public étranger et, selon Nesselrath (p. 121), les trois discours pourraient appartenir au même séjour macédonien. Ces différents éléments permettent de valider leur étude, datation peut-être proche, public

${ }^{20}$ Cf. Branham, op. cit., p. 237-243.

${ }^{21}$ Cf. Schwartz, op. cit., p. 148. Voir également pour les problèmes de datation, Jones, op. cit., p. 168: Herod. appears to be young; p. 169: Lucian apparently young ainsi que Schmid, op. cit., p. 297-319. J. Bompaire relève dans le t. I de l'édition de la CUF (p. XVII) qu'il est en général impossible de classer chronologiquement l'ensemble de l'auvre de Lucien et [que] l'on se contente de certains points de repère fixes, en particulier pour les productions de la vieillesse. MaisJ. Schwatrz a présenté une synthèse intéressante surce problème difficile. 
peut-être semblable. Ceci explique aussi l'exclusion du dernier discours qui aurait pu prendre sa place dans l'analyse qui suit, le De electro, qu'il n'est pas vraisemblable de considérer comme tenu devant un public de Macédoniens, et qui n'appartient pas à la même période.

Par ailleurs G. Anderson, dans son court article paru dans Philologus, 121, a déjà établi des parallèles de construction entre Herodotus et Scytha, analyse qu'il convient d'approfondir et réorienter grâce aux apports du troisième discours, Harmonides.

L'enquête qui suit procédera par comparaisons et allers-retours entre ces trois prolaliai, sans s'interdire des incursions dans d'autres discours, pour tenter de voir, à travers l'écriture et la composition, comment Lucien met en œuvre des recettes qui seraient propres à ce type de discours, tout en individualisant chacun d'eux ainsi qu'il convient à tout rhéteur. C'est par conséquent le versant rhétorique de l'éducation de Lucien qui sera mis à contribution.

Lanalyse se déroulera en deux étapes: déterminer quels sont les points communs entre les trois discours, puis, dans une partie antithétique, considérer quelles sont les spécificités propres à chaque prolalia, autrement dit, mettre en lumière ce qui relève de la richesse créative du sophiste.

\section{Des traits de similitude dans l'écriture des prolaliai}

Indéniablement les trois discours, et plus globalement sans aucun doute l'ensemble des prolaliai, comportent des points de contact, essentiellement dans certaines thématiques, dans leur composition et dans certaines caractéristiques d'écriture.

\section{Des thématiques communes Un portrait de l'orateur}

Si l'on observe nos prolaliai, on détecte systématiquement un même schéma: emploi de procédés rhétoriques (narrations, ékphraseis, éloge etc.) pour mettre en relief la difficulté de la tâche qui attend l'orateur (thème de l'adunaton), et surtout, pour établir un parallèle entre les récits et la situation de Lucien.

Dans Herodotus la presque totalité des $₫ 7-8$ est consacrée à la situation de l'orateur devant le public macédonien, avec les protestations d'usage sur son infériorité par rapport à Hérodote. Le parallèle entre Lucien et Hérodote commence de la façon suivante: "Quant à moi [...] 
j'affirme que je suis bien dans la même situation que lui", '

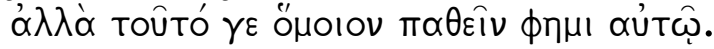

Dans Scytha, même schéma, avec, à partir du \ 9 l'adresse au

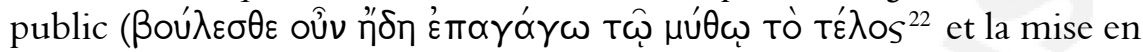
rapport entre l'histoire des Scythes et la situation de l'orateur: "J'affirme que je suis moi aussi dans une situation à vrai dire semblable à celle

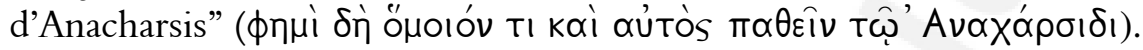

L'histoire du joueur de flûte Harmonide est, quant à elle, mise en parallèle avec celle de Lucien au $₫ 3$ : son exemple s'applique à tous ceux qui sont avides de renommée ( $\delta o^{\prime} \xi \alpha$ ) et en particulier à Lucien, "lors donc que j'eus moi-même formé un dessein semblable au sien et cherché le moyen de me faire connaître le plus rapidement par tous..., "ह $\gamma \omega \gamma$ ' oûv

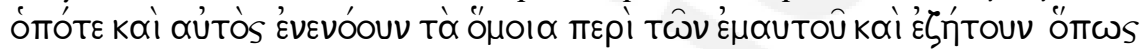

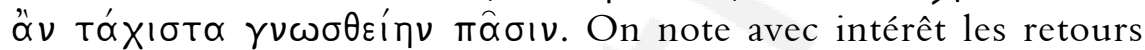

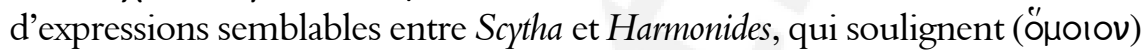
le désir de l'orateur de se comparer aux exemples illustres: kai aútòs;

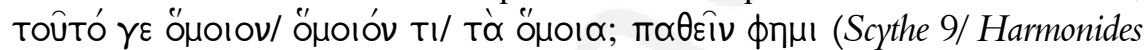
3). On pourrait dire la même chose, mutatis mutandis, de De electro, ${ }^{23}$ autre prolalia tenue devant un public étranger. Si nous nous en tenons à ces quatre prolaliai, on constate que Lucien emploie des "techniques" bien rôdées, vraisemblablement enseignées dans les écoles de rhétorique.

\section{L'éloge de la paideia}

Le $\int 8$ de Herodotus met l'accent sur l'opposition entre le public

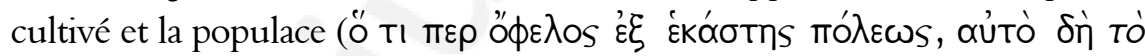

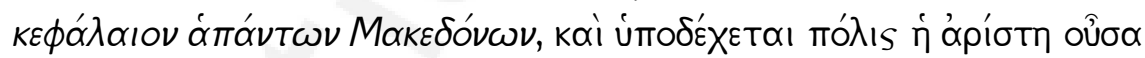

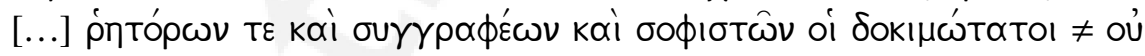

\footnotetext{
${ }^{22}$ A noter une formule semblable pour aborder la partie consacrée au discours de

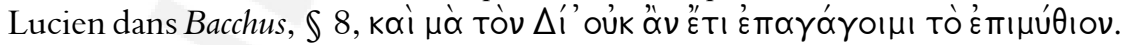

${ }^{23}$ Les traductions de Bacchus, Hercules et De electro sont celles de Bompaire, CUF, T. I. Pour les autres discours de Lucien, les traductions sont celles de Chambry, op. cit., parfois corrigées. Les formulations sont différentes dans De electro même si le sens général est identique $(\mathbb{S} 6)$ : apparition de la première personne du singulier, références à l'auditoire ("Aussi ai-je peur moi-même aujourd'hui à mon sujet: vous qui venez d'arriver et qui nous aurez entendu pour la première fois dans cette

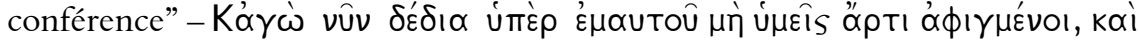

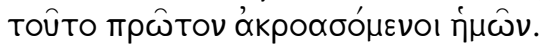




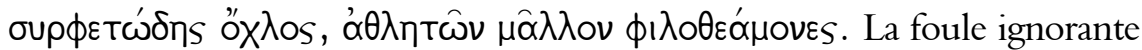
est qualifiée par le terme habituel öx入os, tandis que les pepaideumenoi qui figurent dans le public de Lucien sont caractérisés par leur aristie. La qualité essentielle de ces gens de bien, c'est leur culture, qui s'oppose à l'ignorance de la foule, "avide surtout de voir des athlètes et dont la majorité tient Hérodote pour accessoire" (Herodotus $\ 8$ ). Ainsi les deux notables loués dans Scytha sont-ils de ceux que "pour la science et la puissance oratoire on

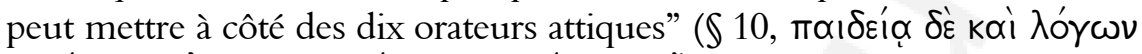

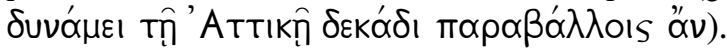

Cet éloge de la paideia est à mettre en lien direct avec l'ensemble de ce discours, puisque Toxaris et Anacharsis viennent à Athènes attirés par la paideia grecque: Toxaris est un sage (бoфós) et "amoureux du beau" ( $\phi 1 \lambda o ́$

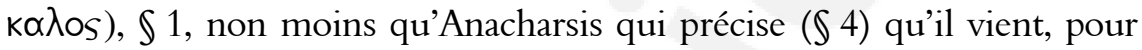
les mêmes raisons, découvrir les plus belles choses de la Grèce. Cet écho entre le début du discours et la fin n'est évidemment pas fortuit, et signale au contraire une volonté qui apparaît clairement dans l'éloge, la mise en parallèle entre les Scythes à la recherche de la culture grecque - et rencontrant Solon, le sage -, et Lucien à qui on recommande deux personnages remarquables par leur paideia. La situation n'est pas différente dans Harmonides, puisque le personnage loué est le meilleur juge $\varepsilon v$

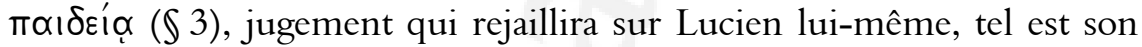

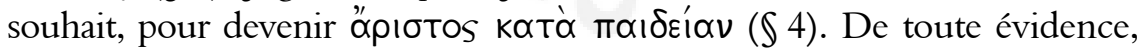
les trois prolaliai, et leur partie encomiastique en particulier, sont d'abord et avant tout un éloge de la culture grecque.

\section{Une captatio benevolentiae: l'éloge du public}

Les prolaliai ont comme fonction essentielle d'annoncer un discours à venir, en développant une captatio benevolentiae: l'orateur va, par conséquent, mettre en œuvre tout l'arsenal rhétorique pour s'attirer les bonnes grâces du public.

La captatio pouvait exiger de la part de l'orateur un éloge, parfois même exagéré, de ses auditeurs, ou d'une partie d'entre eux, plus précisément des notables qu'il convenait de flatter. Lors d'une dialexis il était de bon ton de louer la cité qui vous accueillait, ainsi que les pepaideumenoi qui faisaient l'honneur d'assister à la prestation. ${ }^{24}$

\footnotetext{
${ }^{24}$ La prolalie, à son tour, après la lalia, se rapproche du genre épidictique. Elle n'est plus seulement un exorde ou une "captatio", mais, dans bien des cas, elle devient un petit compliment (cf. Pernot, op. cit., 1993, p. 562).
} 
N'oublions pas qu'il s'agit de discours tenus en Macédoine, c'est-àdire à l'étranger, et que de tels discours sont dûment répertoriés par

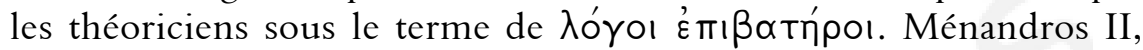
dans la partie qui leur est consacrée $(377,31-378,3)$, précise qu'il convient d'adresser des paroles soit à la patrie qu'on a quittée, soit à la cité qui vous accueille, soit au gouverneur de cette cité.

Dans Herodotus on trouve à partir du $₫ 8$ l'éloge du public et de la cité. Dans Scytha ( $\$ 10$ ss.) on retrouve l'éloge, mais cette fois essentiellement adressé à deux notables, le père et le fils, tandis que Harmonides offre la même louange, mais pour un seul personnage, bienfaiteur de la Syrie, aux \$3-4.

Ces passages ont parfois paru d'excessives flatteries ${ }^{25}$ indignes d'un orateur confirmé. Ce qui peut nous choquer en tant que modernes ne provoquait sans doute pas la même réaction des contemporains de Lucien, pour lesquels se placer sous la protection d'un personnage important ne constituait pas une faute de goût. Il faut cependant noter que l'on ne retrouve pas, dans les autres prolaliai du corpus lucianesque de tels morceaux, aussi insistants. Ce point militerait pour des discours datant plutôt du début de la carrière, quand de tels appuis étaient encore indispensables, ce qu'ils ne seront plus chez un orateur confirmé. Ainsi, aussi bien dans De electro que dans Bacchus ou Hercules de telles insertions sont-elles plus discrètes et plus subtiles.

Quoi qu'il en soit, l'adresse à l'auditoire fait partie intégrante de ces courtes pièces et ces passages seront étudiés plus en détail dans la deuxième partie.

A ce stade de la réflexion, il faut donc relever que les prolaliai qui nous intéressent comportent toutes, une adresse au public, un parallèle avec l'auteur, - traité de diverses manières -, et la mise en scène d'une histoire, ou de plusieurs, destinées, soit à annoncer la lecture ou récitation qui suit, soit à mettre en valeur l'orateur. ${ }^{26}$ Voyons à présent comment se répartissent ces parties.

\footnotetext{
${ }^{25}$ Cf. Nesselrath, op. cit., p. 121: In all three of these introductions - "Herodotus", "Harmonides", "Scytha" - Lucian comes across as still very much an apprentice who is for the first time confronted with the task of winning a friendly constituency and benevolent backers.

${ }^{26}$ On retrouve en partie les préceptes d'Aristote, Rhétorique 1414 b 5-8 concernant les prologues épidictiques.
} 


\section{Une composition stéréotypée?}

"Lucien applique ici les méthodes scolaires et il est inutile de chercher un plan dans ses laliai", affirme Bompaire, p. 287. La remarque semble contradictoire, car si Lucien applique des méthodes scolaires, ce qui est en partie vraisemblable, il est plus que probable qu'il y ait une structure, ce que précisément on apprenait aussi à l'école du rhéteur. Ce n'est en réalité pas tant l'absence de composition qu'il faut noter qu'une certaine liberté de composition. Celle-ci est d'ailleurs inscrite en partie dans les préceptes de Ménandros II, en différents endroits pour les laliai et les prolaliai (391, 19-28; 392, 9-14; 393, 23-24). Ces consignes ne sont pas synonymes de désorganisation totale, mais soulignent la latitude qu'a l'orateur de disposer à son gré certains éléments sans être tenu de suivre un ordre canonique: par exemple pour l'éloge, il reste libre, dans une prolalia, de suivre ou non l'ordre les topoi prévus par les manuels.

Cette liberté de composition se ressent dans plusieurs aspects des trois prolaliai de Lucien.

Mais, avant tout, reprenons la structure des ces récits d'après l'analyse généralement retenue par les commentateurs, que nous serons amenés à rectifier au cours de l'étude:

\begin{tabular}{|c|c|c|}
\hline Herodotus & Scytha & Harmonides \\
\hline 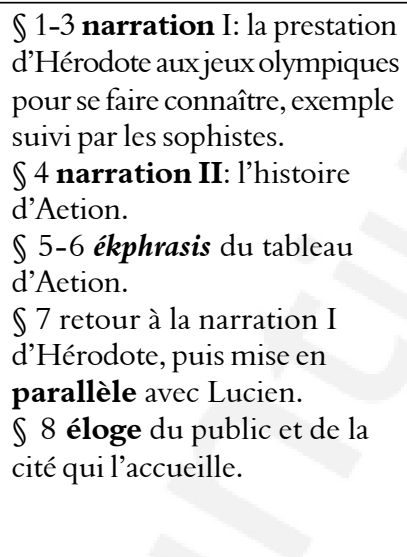 & 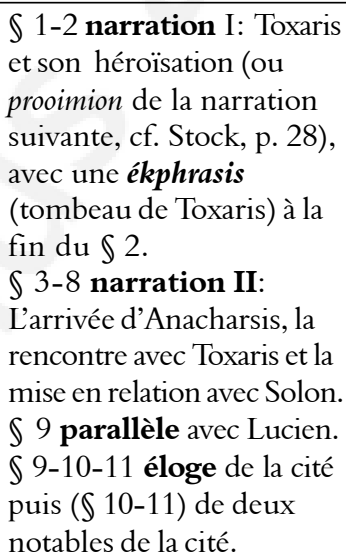 & 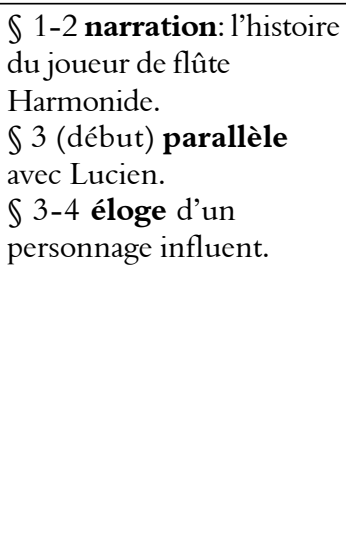 \\
\hline
\end{tabular}

On retrouve des similitudes incontestables dans les trois prolaliai qui débutent par ce qui peut être analysé comme une narration, ${ }^{27}$

\footnotetext{
${ }^{27} \mathrm{La}$ situation est différente par exemple pour Zeuxis, puisque la prolalia commence par les éloges qui sont faits à Lucien pour l'originalité de ses discours, élément qu'il reproche à ses interlocuteurs. Le texte commence donc en réalité par un blâme.
} 
proposent un parallèle avec l'orateur, pour se terminer par un éloge. Les parallèles semblent en particuliers incontestables entre Herodotus et Scytha, avec deux narrations, et une ékphrasis intercalée dans l'une de ces narrations. On ne peut donc pas prétendre y observer une absence de composition, celle-ci, dans les trois exemples apparaissant avec netteté, même si les dispositions de chaque partie et leur longueur proportionnelle peut varier de l'un à l'autre. Il faut avouer également que pour Harmonides la subdivision proposée entre le parallèle de Lucien et l'éloge du notable est plus factice que réelle, dans la mesure où les deux éléments sont étroitement liés dans ce discours, bien plus que dans les deux autres.

Si nous nous intéressons aux articulations qui permettent de passer d'une partie à l'autre, on constate que, contrairement à ce qui a pu être avancé parfois, Lucien use de toute la panoplie rhétorique.

\section{Les transitions}

\begin{tabular}{|c|c|c|}
\hline Herodotus & Scytha & Harmonides \\
\hline 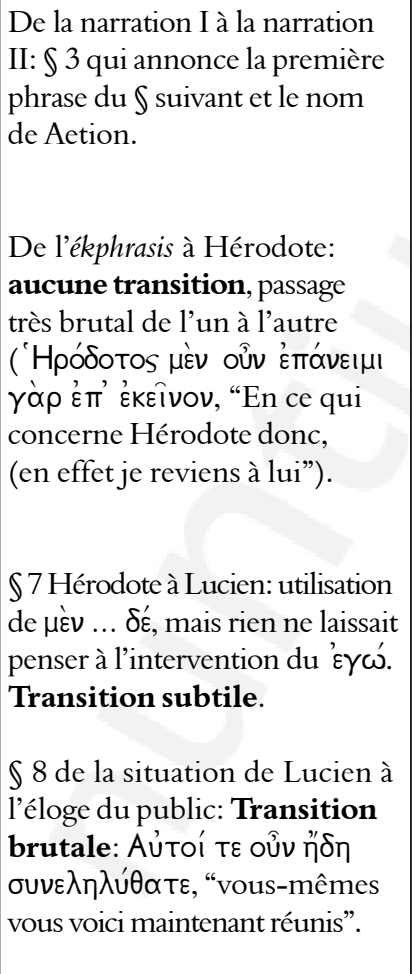 & 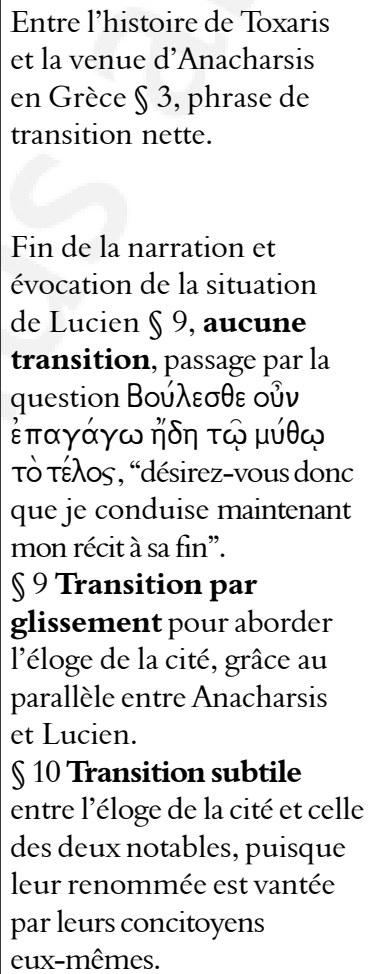 & $\begin{array}{l}\text { Entre la narration et le } \\
\text { parallèle avec Lucien et } \\
\text { l'éloge du notable } \ 3 \text { : } \\
\text { première phrase du } \int \text { forme } \\
\text { transition (rappelle le début } \\
\text { du } \$ 4 \text { de Hérodote). }\end{array}$ \\
\hline
\end{tabular}


Lucien joue de tous les registres pour passer d'une partie à l'autre: par moments, il raffine à l'extrême ses transitions, pour éviter toute brutalité, à d'autres moments, à l'inverse, il semble totalement les négliger. A l'école de rhétorique on apprenait particulièrement à soigner ces passages, aussi peut-on être intrigué par ce qui pourrait apparaître comme une forme de désinvolture, ici ou là (en particulier dans plusieurs passages de Herodotus, $\int \mathbb{S} 7$ et 8 ou au $\$ 9$ du Scytha), dans la brutalité du passage d'une partie à l'autre. Soit on considère qu'on a les signes d'écriture d'un orateur inexpérimenté, soit on doit se demander s'il ne s'agit pas d'une volonté délibérée. Cela pourrait même être la marque des prolaliai et de cette liberté de composition qui confere à ces discours une tonalité si particulière de légèreté. Ces ruptures apparentes, en réalité, tout en mimant la spontanéité, et par conséquent une certaine fraîcheur, sont le fruit de l'art consommé de l'orateur. ${ }^{28}$ Cette variété que l'on peut repérer dans les transitions, rejoint une des caractéristiques d'écriture des prolaliai, discours entièrement tournés vers l'agrément et le charme.

\section{Une écriture plaisante et enjouée}

A l'opposé du ton sérieux de la mélétê qui la suit souvent, la prolalie adopte un ton plus léger, propre à disposer favorablement l'auditeur. Le style doit être plein de charme (í̉ovń; hédoné) ainsi que le précise Ménandros II pour les laliai $(389,12,15-16$ = iotopías jííotas, 28), en prenant comme exemples, soit des récits qui mettent en scène des dieux ou des héros, 389, 16-22 (cf. chez Lucien par ex. Bacchus, Hercules), ou des récits semblables à ceux d'Hérodote, 389, 27 ss. ('Hробótou

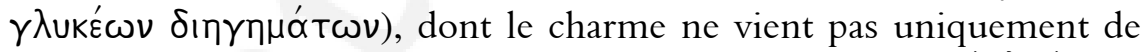

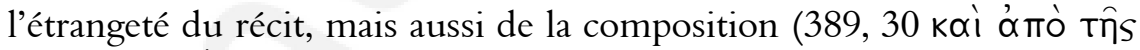

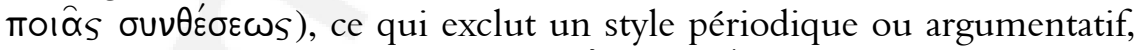

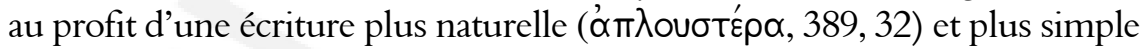
( $\left.\propto \phi \varepsilon \lambda \varepsilon \sigma \tau \tau^{\prime} \rho \alpha\right)$. Pour parvenir au résultat escompté, charmer l'auditoire,

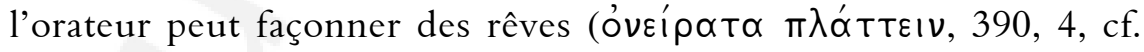
Somnium) ou prétendre rapporter des propos (De electro). Les

\footnotetext{
${ }^{28}$ Comme l'a bien vu L. Pernot, op. cit., 1993, p. 563: Les prolalies de Lucien, tout en étant composées avec une parfaite netteté, évitent de justifier l'enchaînement des différentes parties et préfêrent les attaques abruptes, les transitions arbitraires. [...] La liberté de composition est un effet de l'art.
} 
protestations de véracité que l'on peut noter dans les trois prolaliai ressortissent exactement de cette esthétique prônée par Ménandros; ainsi, au début de l'ékphrasis du tableau d'Aétion, Lucien va-t-il prétendre l'avoir vu (\$5), tandis que l'éloge des deux notables dans le Scythe s'achève sur la véracité de la rumeur publique attestée par un serment du plus plaisant effet $(\mathbb{S} 11$, "Voilà ce que j'ai entendu dire à tout le monde, j'en jure par Zeus, s'il faut ajouter encore un serment à ma

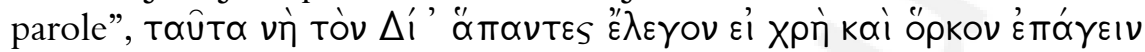

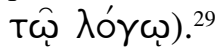

Ménandros II évoque en outre, à plusieurs reprises, la $\gamma \lambda u k u ́ t \eta s$ (glukutês), la douceur, nécessaire à ces discours (389, 14, 27). Les termes

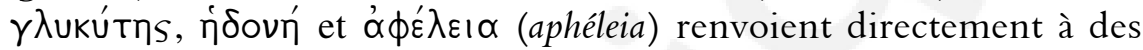
catégories stylistiques dûment recensées par Hermogène le Rhéteur

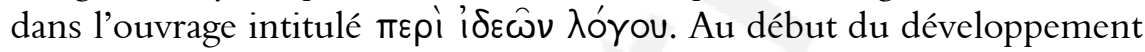
sur la glukutês cette dernière est nommément liée à l'hêdonê $(330,1)$, les deux se trouvant essentiellement dans les pensées mythiques, ${ }^{30}$ ce qu'on retrouve par exemple dans les noces d'Alexandre et Roxane, ou encore dans le récit, à la frontière de la légende, de Toxaris puis d'Anacharsis. Il n'est pas jusqu'aux récits d'Hérodote aux jeux olympiques, ou d'Harmonide qui ne comportent également cet élément. En effet, Hermogène précise qu'après les discours mythiques, sont aussi concernés par le plaisant "les récits proches du mythe, comme de narrer les événements de la guerre de Troie [...] puis viennent les récits qui par petits bouts en quelque sorte rejoignent le mythe, mais auxquels on accorde plus de créance qu'aux mythes, comme c'est le cas de tous les récits d'Hérodote". ${ }^{31}$ Dernière catégorie de discours plaisant, "tout ce qui procure un plaisir à nos sens, je parle de la vue, du toucher, du goût ou de toute autre jouissance", peut-être plus encore que les récits mythiques. ${ }^{32}$ Difficile de ne pas rapprocher avec les ékphraseis qui, parlant à notre vue, s'inscrivent clairement dans cette esthétique. Hermogène

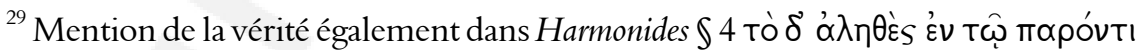
$\delta \varepsilon ı \chi \forall \dot{\sigma} \sigma \varepsilon т \alpha ı$.

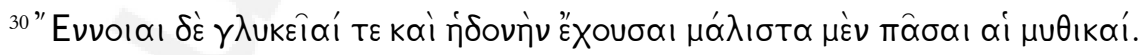

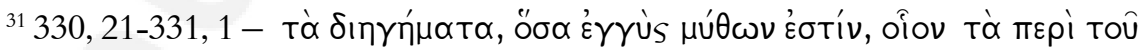

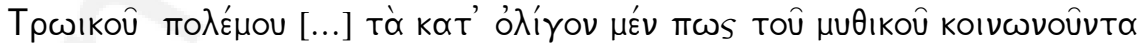

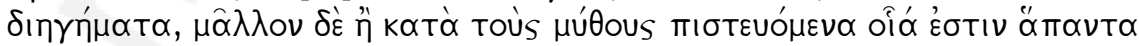
та̀ 'Hрodótou.

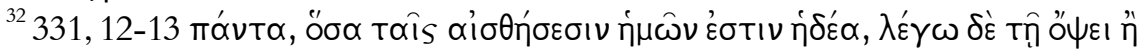

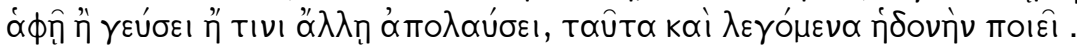


souligne ensuite que l'expression poétique procure également de la glukutês, mais seul Scytha, parmi les trois prolaliai, intègre des citations poétiques ( $\$ 9$ et 11$)$.

La catégorie de l'aphéleia, la simplicité, est, dans sa méthode et son expression, semblable à celle de la pureté ( $k \propto \theta \propto \rho o ́ t \eta s ~ k a t h a r o t e ̂ s)$, cf. 327, 24 et 328, 15-16, et, en règle générale, doit éviter toute complication. Ainsi relève de la katharotês "la présentation des faits sur

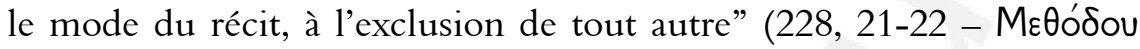

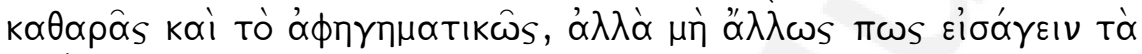
пра́ $\gamma \mu \alpha \tau \alpha)$. Autrement dit, Lucien, en choisissant délibérément le mode narratif pour ses prolaliai s'inscrit dans la démarche de la katharotês et de l'aphéleia, surtout en évitant toute digression et en s'en tenant, comme le dit Hermogène $(227,20)$ au fait brut sans rien y adjoindre.

Au niveau de l'expression l'aphéleia se manifestera essentiellement par des kôla brefs, l'absence de tournures obliques, à savoir des propositions participiales (comme le génitif absolu par ex.) et de périodes trop longues et complexes. Il n'est pas possible, dans le cadre de cet exposé, d'analyser en détail ces trois discours sous l'angle stylistique. Prenons comme exemple le début de Scytha: Oûtos ó Tógapıs oủdè

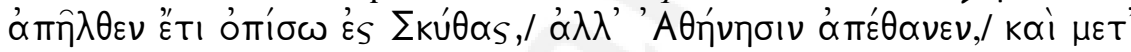

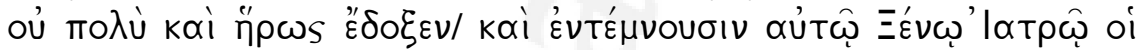

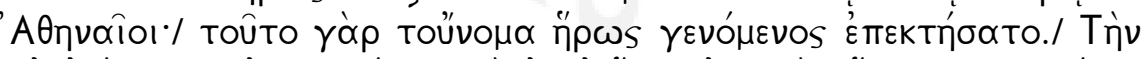

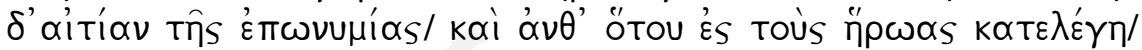

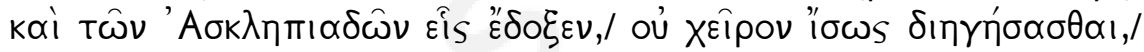

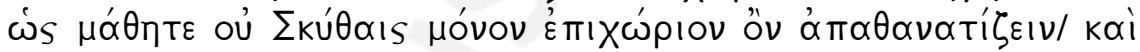

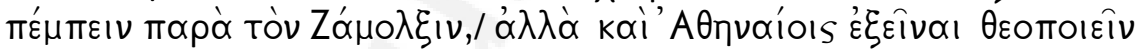

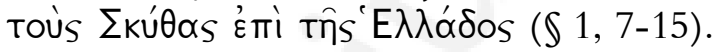

Les barres obliques séparent les kôla. On constate que jusqu'à

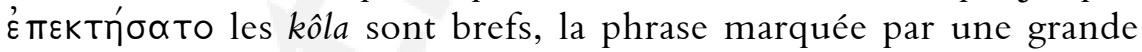
simplicité, sans subordination, c'est essentiellement le kớ qui assure les liens, nous sommes sans conteste dans une ecriture caractérisée par l'aphéleia. A partir de Tìv $\delta$ 'aíríav la situation est un peu différente, dans la mesure où la phrase est plus tortueuse, avec un complément au début, dont le verbe n'apparaît qu'une ligne plus loin (oủ Xદipov íows

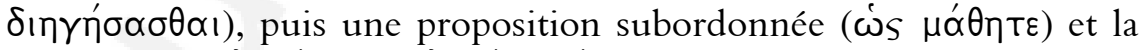

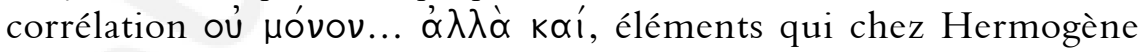
s'apparentent bien davantage à l'ampleur (peribolê) et à la grandeur (mégethos). Indéniablement dans le début du passage on a un style simple, alors que dans le second il l'est beaucoup moins. Il faudrait donc faire une analyse systématique des discours pour avoir une idée plus précise de l'écriture adoptée par Lucien. Mais on sent bien à la lecture, que les 
discours, dans l'ensemble, n'offraient aucune difficulté de compréhension pour l'auditeur, le vocabulaire est courant, la syntaxe coulante. Ce que nous enseignent les observations ci-dessus, c'est que, d'abord, Lucien a mis en œuvre ce style simple, tonalité dominante, même s'il est difficile de dire dans quelles proportions, ensuite, que, bien entendu, un discours mêle généralement étroitement plusieurs traits d'écriture, sans quoi il court le risque de devenir rébarbatif. Ce qui est certain c'est que la glukutês, par le biais même des récits et des ékphraseis mêlant mythes, légendes, fictions et pseudo-réalités, procurait cette hêdonê, ce charme au discours, dont il convenait, par ailleurs, qu'il soit rédigé dans une langue simple et facilement accessible.

Cela n'empêchait ni le trait d'humour, ni la finesse (ózútns,

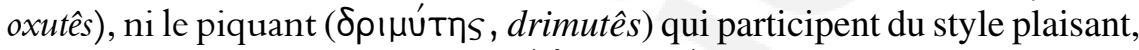

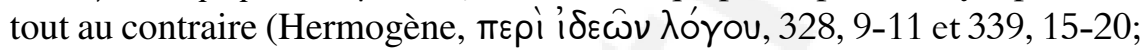
Ménandros II, 388, 28-29). On peut noter que Lucien ne se prive pas d'exploiter une veine humoristique, généralement à son propre détriment, dans une sorte d'autodérision $/ / \$ 8$ Bacchus). C'est de cette façon certainement qu'il convient d'entendre la prétention d'être perçu

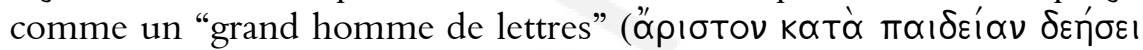

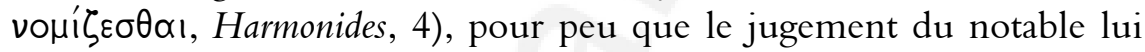
soit favorable. L'humour ne manque d'ailleurs pas dans ce court récit, en particulier dans la narration de la fin tragique d'Harmonide, qui, au moment où il allait enfin réaliser son rêve - connaître le succès et devenir célèbre -, ambition de Lucien également, mourut. La manière qu'a Lucien de raconter cet épisode tragique est plein d'enjouement et de saveur: "Harmonide n'eut pas le temps de profiter de ces conseils. On dit qu'au premier concours où il joua, il se laissa emporter à l'amour de la gloire et souffla si fort qu'il rendit le dernier soupir dans sa flûte et mourut sur la scène sans avoir obtenu la couronne. Ce fut la première

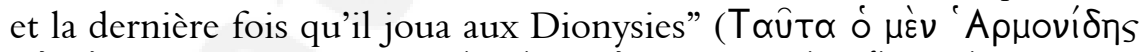

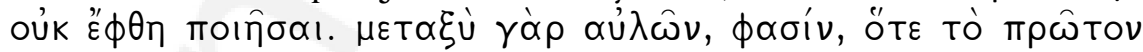

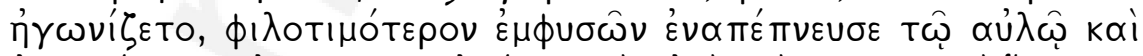
๔oт

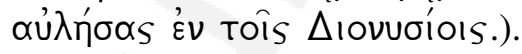

Une étude stylistique affinée de ces discours reste à faire, mais il est une chose assurée, car Lucien lui-même insiste sur ce point, c'est le souci de l'écriture qui fut le sien. Il suffit de relire le début de Zeuxis, ou d'entendre les éloges du style d'Hérodote (Herodotus, 1), voire les paroles adressées par Harmonide à son maître $(\mathbb{S} 1)$, dans lesquelles il utilise, pour parler de la maîtrise de la flûte, des termes proches de ceux utilisés pour l'écriture. 


\section{Des traits propres à chaque discours L'importance des exercices scolaires}

Comme cela a été relevé, ${ }^{33}$ la méthode de Lucien dans les prolaliai "est fondée très largement sur les exercices scolaires". Il intègre dans chacune de ses prolalies un, voire plusieurs éléments suivants: narration

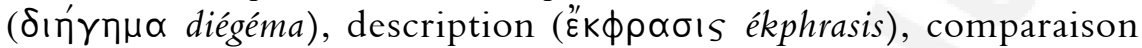

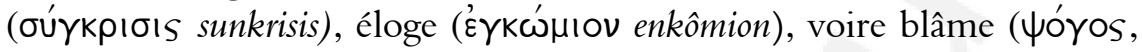
psogos, dans Zeuxis 1-2), liste à laquelle il convient sans doute d'ajouter, comme on le verra, la chrie (Xpeí $\alpha$ ). Ces exercices appartiennent tous à la liste des progymnasmata, travaux proposés aux élèves de rhétorique, liste canonique qui apparaît constituée à partir du $\mathrm{I}^{\mathrm{er}}$ siècle après J.-C., chez Quintilien et Théon.

Les trois prolaliai comporteraient toutes, comme nous l'avons vu dans la première partie, une narration, un éloge, des parallèles, et, dans Herodotus et Scytha seulement, une ékphrasis. Cependant, les proportions de chacune des parties, et donc, la construction d'ensemble, ne sont pas les mêmes.

\begin{tabular}{|c|c|c|}
\hline $\begin{array}{c}\text { Herodotus } \\
\text { (total de } 937 \text { mots) }\end{array}$ & $\begin{array}{c}\text { Scytha } \\
\text { (total de } 1850 \text { mots) }\end{array}$ & $\begin{array}{c}\text { Harmonides } \\
\text { (total de } 988 \text { mots) }\end{array}$ \\
\hline $\begin{array}{l}\text { Narration }=\$ 1-3(369 \text { mots }) \\
\text { Ékphrasis }=\S 4-6(322 \text { mots }) \\
\text { Narration }=7 \text { (26 mots })\end{array}$ & $\begin{array}{l}\text { Narration }=\$ 1-8(1191 \text { mots }) \\
\text { Ékphrasis }=\text { fin } \ 2(110 \text { mots })\end{array}$ & Narration $=1-2(567$ mots $)$ \\
\hline $\begin{array}{l}\text { Situation de Lucien }=\text { fin } \\
\text { du } \$ 7 \text { (93 mots) }\end{array}$ & $\begin{array}{l}\text { Situation de Lucien }=\text { début } \\
\text { du } ₫ 9 \text { (88 mots) }\end{array}$ & $\begin{array}{l}\text { Situation de Lucien }= \\
\text { (début } ₫ 3)(69 \text { mots) }\end{array}$ \\
\hline $\begin{array}{l}\text { Éloge de la cité et du public } \\
=\int 8 \text { (124 mots) }\end{array}$ & $\begin{array}{l}\text { Éloge de la cité et des deux } \\
\text { notables }=9 \text { (fin)-11 (470 mots) }\end{array}$ & Éloge $=3-4$ (350 mots) \\
\hline
\end{tabular}

Ces évaluations sont relatives, dans la mesure où il n'est pas toujours aisé de déterminer ou commence l'éloge: par ex. dans Scytha se trouvent mêlés au $\ 9$ la situation de Lucien et un éloge de la cité qui l'accueille. Cependant quelques observations s'imposent: dans Harmonides, et dans une moindre mesure dans Scytha, les éloges qui ponctuent ces discours sont très développés, beaucoup plus que dans Herodotus. Plus d'un tiers du total pour Harmonides, et environ un quart pour Scytha. Cela s'explique assez facilement par la différence de l'objet de l'enkômion, dans un cas (Herodotus) un groupe indistinct de citoyens formant l'auditoire érudit de Lucien, dans l'autre, des personnalités pour lesquelles il est nécessaire de développer un éloge individuel.

${ }^{33}$ Voir en particulier Anderson, op. cit.; Reardon, op. cit., p. 165. 
On peut constater, également, que les narrations, dans Herodotus et Scytha, sont de longueur à peu près équivalente, mais que l'ékphrasis dans le premier discours représente environ un quart du récit, alors qu'elle compte pour moins d'un dixième dans Scytha. Dans les deux cas, par contre, l'ékphrasis se trouve enchâssée dans la narration. La structure plus équilibrée de Herodotus confirme que le retour brutal, sans transition, du personnage d'Hérodote, au $\$ 7$, n'est vraisemblablement pas une maladresse de jeune débutant, mais au contraire un travail d'orateur soucieux d'une construction soignée.

\section{Le récit $(\delta ı \eta ́ r \eta \mu \alpha)$}

Tous les traités de progymnasmata en notre possession comportent

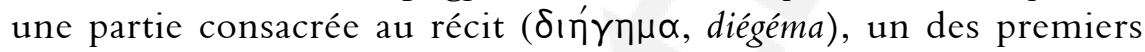
exercices de l'apprenti rhéteur. Ils le décrivent comme un discours qui expose des faits réels, ou donnés comme tels; ${ }^{34}$ la vraisemblance apparaît comme un des traits essentiels, avec la clarté et la concision (Théon, 79, 20 ss.). ${ }^{35}$ La concision est mise en avant par Lucien au $\$ 8$ du Scythe:

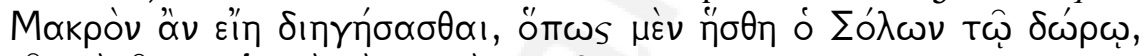

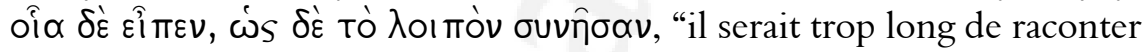
combien ce présent fut agréable à Solon, ce qu'il dit, comment désormais ils vécurent ensemble". Cette phrase joue, sur plusieurs registres, Lucien, par l'emploi du verbe technique $\delta ı \eta \gamma \eta ́ \sigma \alpha \sigma \theta \alpha ı$ soulignant à ses auditeurs cultivés, dans une sorte de connivence, qu'il est en train de développer un récit, dont il connait, comme eux, les impératifs de concision, tout en précisant par la suite, dans une sorte de prétérition, tout ce qu'il prétendait, quelques lignes avant, être trop long à développer!

Lautre élément qui revient constamment chez les technographes, c'est la clarté, qui d'ailleurs est aussi implicitement recommandée pour le style simple, et pour parvenir à l'aphéleia; le Pseudo-Hermogène recommande ce qu'il nomme la figure directe ("la figure directe convient

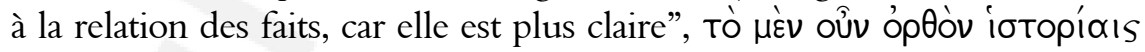

\footnotetext{
${ }^{34}$ Les quatre principaux recueils de progymnasmata, celui de Théon, du pseudoHermogène, d'Aphthonios et de Nikolaos de Myra, définissent le récit de cette manière. Les préceptes des recueils de progymasmata s'inspirent assez souvent des théories rhétoriques correspondantes telles qu'on peut les grouver par exemple dans la Rhétorique à Alexandre, dans les traités cicéroniens ou chez Quintilien.

${ }^{35}$ Aphthonios ajoute à ces virtutes narrationis traditionnelles la "correction de la langue".
} 


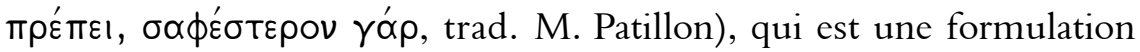
dans laquelle l'énoncé maintient le plus possible le cas nominatif pour évoquer l'action des personnages (ce mode s'oppose par exemple à l'assertive oblique, "on raconte que...").

Le mode décrit par le Pseudo-Hermogène est celui choisi dans les trois cas par Lucien, les personnages respectifs de chaque narration étant sujets de cette dernière. D'autre part, les trois discours commencent par le nom du personnage principal concerné par la narration ( $A \rho \mu o v i \delta n s$

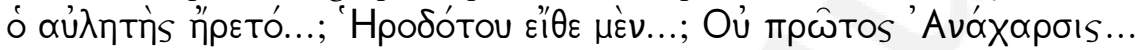

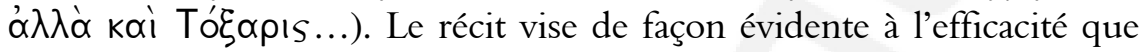
lui donne un rythme rapide, et une entame directe.

Les anecdotes sont particulièrement bien choisies par rapport à un objectif qui apparaît à la fin des discours, en-dehors du champ du récit: pour Hérodote et Harmonide c'est la recherche de la gloire, pour Toxaris et Anacharsis c'est le désir de connaître la culture grecque ( $\pi \alpha ı \delta \varepsilon i \alpha s$

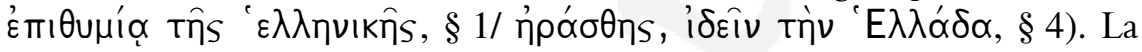
rigueur de la construction au service du but poursuivi par Lucien, se révèle, entre autres, dans les parallèles lexicaux et les effets de miroir entre les différentes parties.

\section{Des occurrences lexicales}

Le notable loué dans la partie finale de Scytha ressemble à Solon (largement mis en scène dans la narration), Périclès ou Aristide (\$11), c'est-à-dire aux meilleurs des Grecs, réputés pour leur sagesse et leur vertu, alors que la prolalia n'a de cesse de mettre en avant l'aristie, avec

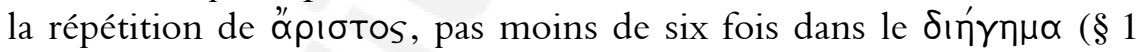

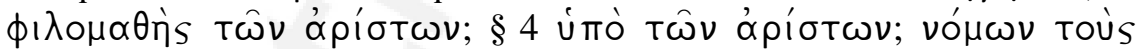

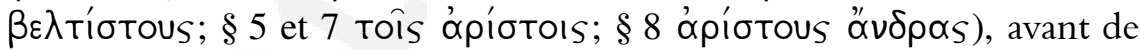

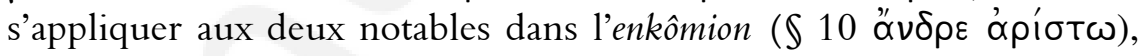
personnages qui ne souhaitent que le mieux pour leur cité $(\mathbb{S} 10$

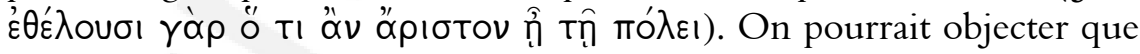
le terme est courant et attendu dans un discours dont le but est l'éloge, mais dans Herodotus par exemple on ne trouve que deux occurrences du superlatif, et dans Harmonides trois, dont une seule dans la narration

\footnotetext{
${ }^{36}$ Opposition fondamentale entre les narrations avec ou sans relais de la parole, cf. M. Patillon, Introduction à l'édition de la CUF des Progymnasmata de Théon, p. XLVI.
} 
( $(2)$. Scytha semble donc, plus que les deux autres, dévolu à ce thème, dans le but de disposer favorablement les deux personnages célébrés dans toute la cité où Lucien s'apprête à faire ses lectures.

Dans les deux autres prolalies d'autres occurrences peuvent être détectées, en particulier des termes ayant trait à la gloire, la célébrité. Celle-ci constitue l'objectif avoué des personnages des récits, Hérodote,

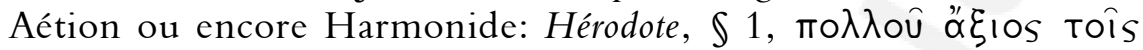

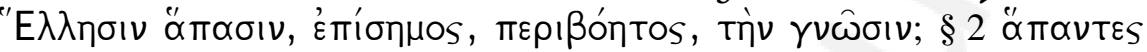

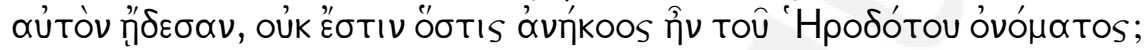

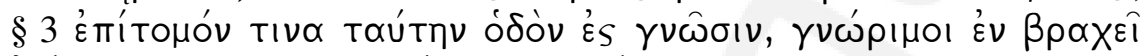

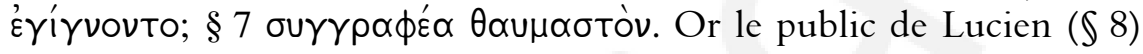
est constitué de "l'élite de chaque cité, [de] la partie la plus importante

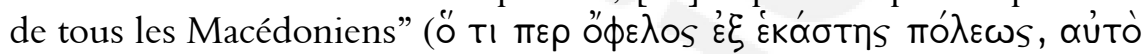

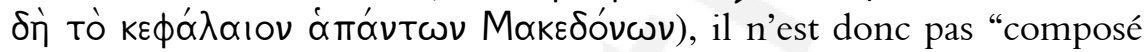

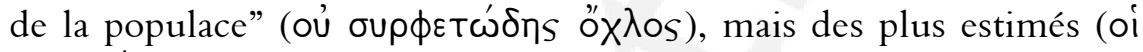

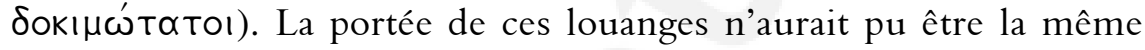
sans la narration qui avait préparé le public à entendre ce final.

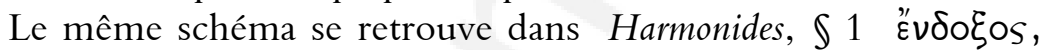

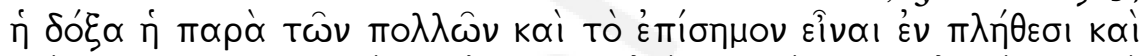

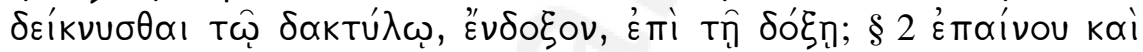

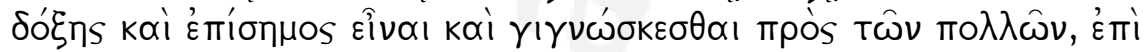

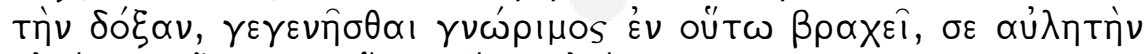

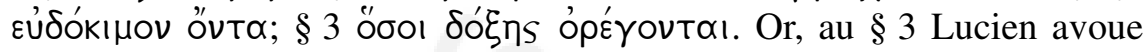

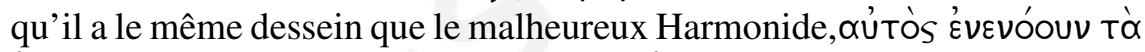

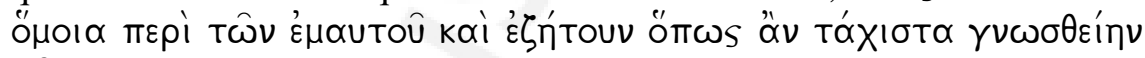
Tốoıv, "moi-même j'avais en tête les même projets pour moi-même et je recherchais comment être connu de tous le plus rapidement possible".

Mais, encore plus intéressantes dans ce discours, sont les occurrences de $\varepsilon^{\prime}$ traıvos, la louange, mot qui scande toute la prolalia, alors qu'on ne le rencontre qu'une fois dans Scytha et jamais dans

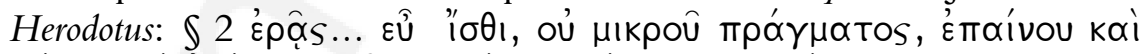

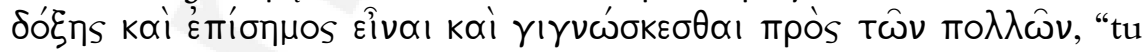
es épris, sache-le bien, d'une chose qui n'est pas petite, de louange, de gloire, et d'être célèbre et d'être connu du grand nombre" tel est l'avertissement que prodigue le maître Timothée à son jeune élève ambitieux, Harmonide, en employant, pour insister sur l'ampleur de la

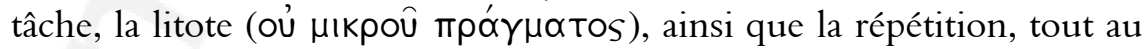

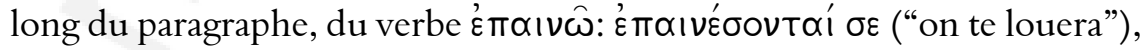
puis un florilège de formes verbales dessinant une sorte de syllogisme,

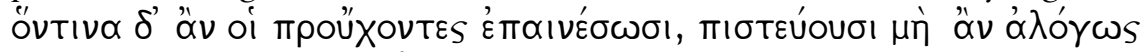

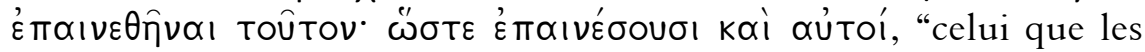


premiers citoyens louent, ils [les gens du peuple] croient que ce n'est pas sans raison qu'il est loué; par conséquent ils le louent à leur tour". Conseil qui peut sembler un tant soit peu sophistique, voire cynique, mais qui rentre par ailleurs dans une problématique constante dans l'Antiquité, la multitude est-elle capable de juger et de distinguer le beau? A la fin du récit, au début du $₫ 3$ qui forme transition, Lucien propose un élargissement du cas d'Harmonide "à tous ceux qui aspirent à la gloire, en se produisant en public, en recherchant l'éloge du plus

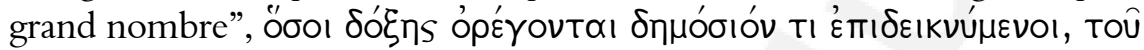

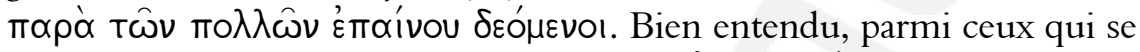

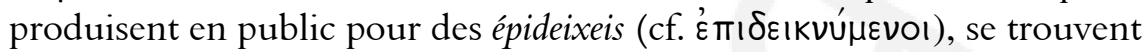

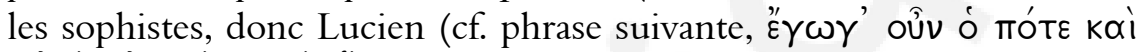

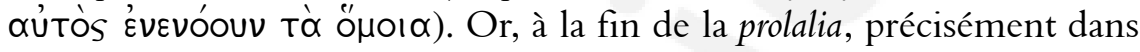
l'éloge du notable dont le jugement doit lui assurer succès ou désespoir, Lucien a ce cri face à son interlocuteur qui tient sa destinée dans son jugement (\$3): "Si je te dévoilais mes productions et si tu les louais, [...] alors je serai parvenu au comble de mes espérances et j'aurai

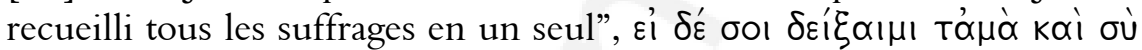

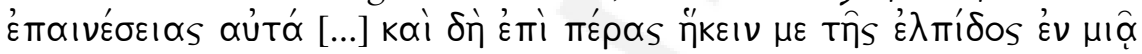

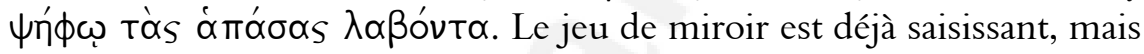
s'accentue encore à la fin du discours avec la réflexion du $\$ 4$, puisque

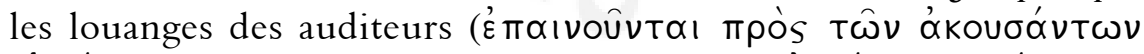

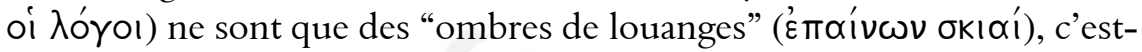
à-dire des louanges mensongères face à la "vérité" (Tò $\delta$ ' $\alpha \lambda \eta \theta \dot{s}$ ) que sera le jugement de ce notable. Après l'évocation de la louange, perçue négativement, de la foule, puis celle, espérée positive, du haut personnage, voici celle des dieux eux-mêmes qui est invoquée dans la dernière phrase: "En effet, puissions-nous sembler, dieux, dignes du discours et confirmez pour nous l'éloge reçu auprès des autres",

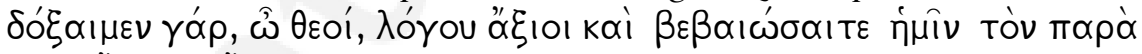

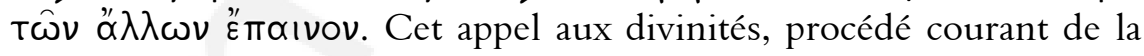
rhétorique, ne manque pas de sonner de manière humoristique dans cette fin de prolalia, mais surtout nous entraîne dans un jeu très sophistiqué, celui de l'éloge du notable qui, occupant plus du tiers du discours, ne semble plus occuper cette place que pour l'obtention d'éloges pour Lucien lui-même, objectif si important que les dieux sont invoqués à la rescousse. Ces allers-retours entre les éloges demandés par Harmonide, ceux prodigués par Lucien à son interlocuteur et qui, en même temps, les sollicite pour lui-même, expliquent l'importance de l'enkômion dans ce discours. Et le maître Timothée avait prévenu qu'il était difficile d'acquérir des éloges. C'est toute la subtilité des 
prolaliai que l'on retrouve ici, cette touche de finesse cachée derrière une apparente simplicité, celle du style et de l'expression, qui permet à l'orateur de se faire entendre à demi-mots, de faire admirer sa prouesse en disant sans dire clairement, d'afficher son talent tout en jouant la modestie.

\section{L'ékphrasis}

L'ékphrasis remplit essentiellement deux rôles bien circonscrits chez les théoriciens, soit le charme et la douceur (Ménandros II 433, 13-16; Hermogène, peri ideôn 331, 14-332, 2), soit l'amplification pathétique (Quintilien VIII, 3, 67-70). ${ }^{37}$ Mais la description ne doit jamais être détachée de son contexte.

Deux de nos prolaliai contiennent une ékphrasis, de longueur très inégale, Herodotus et Scytha. D'ailleurs, le vocabulaire de l'image, eikôn, eikazein, est présent dans les deux discours (Herodotus $\ 7$, Scytha 9). Dans un cas, Herodotus, nous avons une longue description du tableau d'Aétion, dans l'autre, Scytha $\ 1$, celle du tombeau de Toxaris. ${ }^{38}$ Il est nécessaire de s'attarder plus longuement sur l'ékphrasis du tableau d'Aétion.

\section{L'ékphrasis du tableau d'Aétion}

La première question qui se pose, vu la longueur de la description, est celui d'une possible digression à l'intérieur du récit. Or, Théon

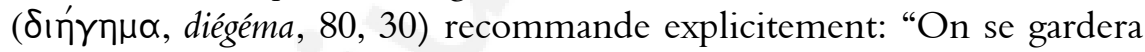
d'intégrer dans le récit de longues digressions..." (trad. M. Patillon). Quelle fonction peut occuper l'ékphrasis à l'intérieur des deux récits, celui d'Hérodote et celui d'Aétion?

La structure d'ensemble est complexe, et particulièrement travaillée: on a un récit 1, celui d'Hérodote qui se produit aux jeux olympiques; puis un récit 2, celui d'Aétion qui exposa son tableau également aux jeux olympiques et put ainsi obtenir la main de la fille de l'un des hellanodices, Proxénide; le récit 2 laisse la place à l'ékphrasis du tableau des noces d'Alexandre et Roxane; le récit 2 reprend ses droits pour une conclusion $(\$ 6)$ de Lucien; le récit 1 se termine par le rappel

\footnotetext{
${ }^{37}$ Cf. Pernot, op. cit., 1993, p. 670 ss.

${ }^{38}$ Cf. Nesselrath, op. cit., p. 121: The first [story] contains a short "ékphrasis” of a funerary monument.
} 
de l'action d'Hérodote (début du \7). L'ékphrasis se trouve par conséquent enclavée à l'intérieur du récit 2, celui d'Aétion, lui-même enclavé dans le récit 1, celui d'Hérodote. Ce dernier apparaît donc, aux oreilles de l'auditeur, comme le récit principal. Quel est par conséquent l'intérêt des paragraphes 4-6? Le deuxième récit ne fait que conforter le récit 1 , mais cela à vrai dire avait déjà été fait par l'intermédiaire du $\$ 3$, (paragraphe de transition) avec la mention des sophistes qui, à l'instar d'Hérodote, vinrent se produire dans les panégyries et acquérir ainsi une grande renommée. Le récit d'Aétion est-il inutile et quel lien peut-on y voir avec le reste de la prolalia?

On peut schématiser cette construction de la façon suivante:

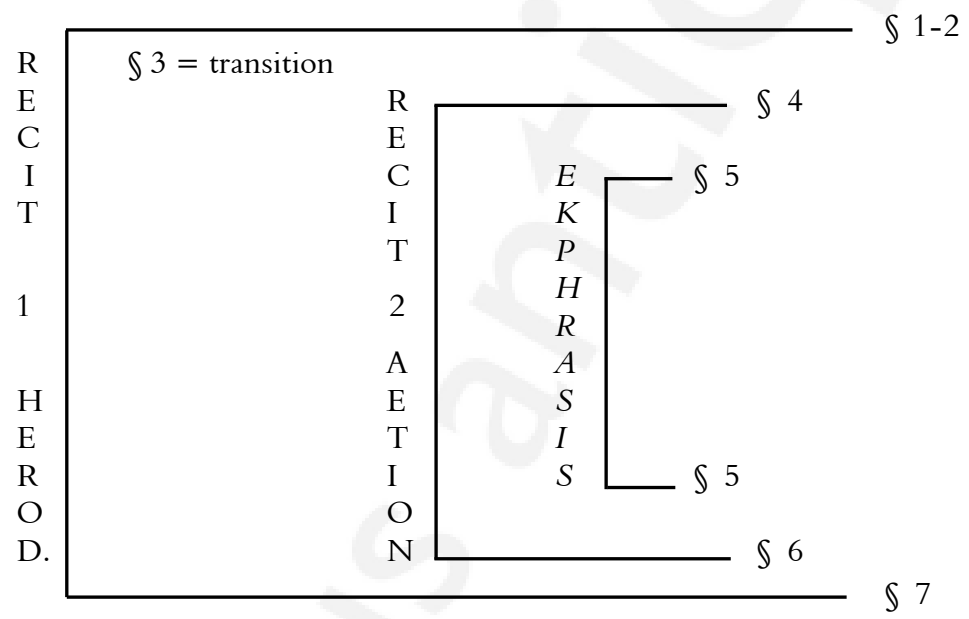

Première remarque: Lucien, en contant l'histoire d'Aétion, ne s'écarte pas de la thématique, Hérodote et Aétion ont suivi la même démarche, dans la même fête, les Jeux Olympiques, et connu le même succès. D'autre part, dans les deux cas, le lien avec l'Histoire est fort, Hérodote est le "père de l'histoire grecque" et le tableau peint par Aétion représente un des grands personnages de l'histoire antique, Alexandre le Grand. Pour autant, les noces d'Alexandre et Roxane n'ont, apparemment, pas grand chose à voir avec la prolalia, si ce n'est qu'Alexandre était Macédonien et que Lucien se produit en Macédoine!

En réalité l'ékphrasis permet à Lucien d'intensifier les effets de miroirs avec le reste du discours, et plus particulièrement avec sa situation personnelle. Tout d'abord, le peintre Aétion est, à l'instar de Lucien en Macédoine, un étranger à Olympie, $\ 4$ oủk $\varepsilon$ mix $\chi \omega p i ́ \omega$. Luimême, d'ailleurs, au $\ 6$ met l'auditeur sur la piste de ces parallèles: "Le peintre s'en retourna marié et son mariage fut comme un accessoire 
de celui d'Alexandre. Il eut le roi pour paranymphe et le prix d'un hymen en peinture fut un hymen véritable". La mise en abyme est évidente: de même que le prix d'une peinture représentant un mariage fut un hymen, de même le prix attendu par l'orateur pour ses narrations et son ékphrasis représentant des triomphes, doit être le succès auprès de son public. Et il attend de ce dernier qu'il joue le rôle de paranymphe, en lui accordant la main de "triomphe", rôle que joua l'hellanodice dans le récit. Ce qui autorise une telle interprétation, ce sont, par ailleurs, les multiples résonances lexicales que l'on retrouve tout au long de la prolalia.

Le réseau lexical est dense entre les trois parties. Le couple

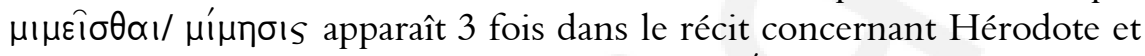
est repris une fois dans le récit d'Aétion ( $\mu$ ıoú $\mu \varepsilon v o$ ), confirmant ainsi que ces deux épisodes sont des modèles à imiter pour Lucien. Or, ce dernier, au $\$ 7$, se hasarde, à travers une sorte de prétérition, à se comparer

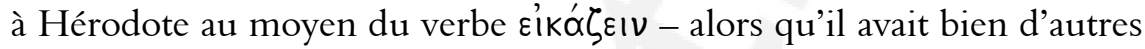
possibilités à sa disposition pour exprimer la comparaison -, verbe qui

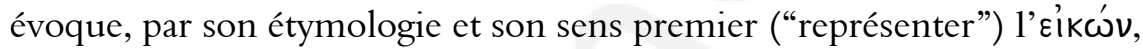
l'image, celle de la représentation peinte par Aétion (\$5), dont l'ékphrasis

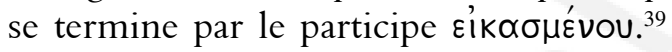

De même, pour évoquer son désir de se faire connaître de tous les Macédoniens, Lucien va curieusement utiliser l'expression ó $\mu \dot{\varepsilon} v$

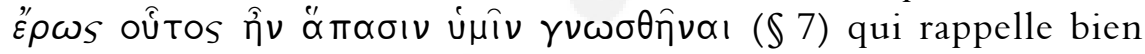
évidemment les Amours décrits dans la peinture d'Aétion. Limpide

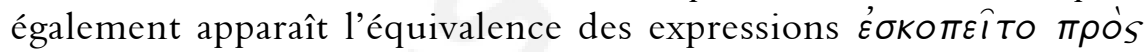

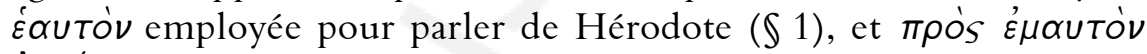

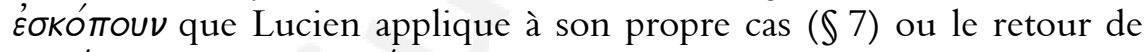

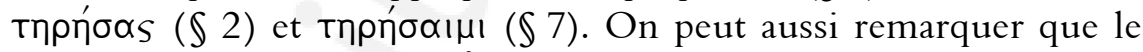
discours commence avec $\varepsilon \dot{X} \chi \hat{\eta} S(\mathbb{S} 1)$, terme qui achève le parallèle

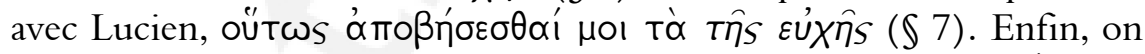
ne peut être insensible à la multiplicité des occurrences de $\delta \varepsilon i ́ k v \cup \mu$,

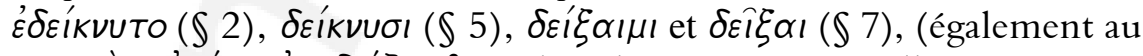

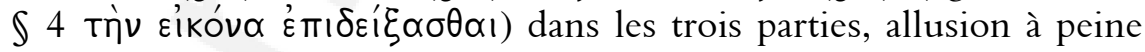
voilée à la démonstration oratoire de Lucien, l'épideixis.

On pourrait faire des constatations analogues avec les deux autres prolaliai. Cela souligne que la description est parfaitement intégrée dans le discours, même si la question de sa présence, voire de son développement, n'est pas totalement résolue.

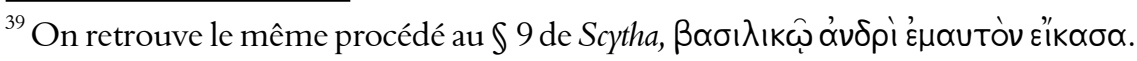


L'articulation entre l'épisode de Hérodote et Aétion apparaît pourtant très clairement dans sa logique: la démarche de l'historien a été copiée, peu après, par les sophistes $(\$ 3)$, précision qui pouvait amener chez l'auditeur une double objection; il s'agit d'exemples anciens, qui ne sont donc plus d'actualité, raison pour laquelle Lucien précise, dans cette expression qui a beaucoup intrigué les savants,

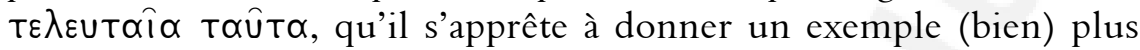
récent (en réalité d'époque hellénistique); l'autre objection, non formulée car sans doute moins importante, c'est celle qui consisterait à limiter les exemples au champ "littéraire". Le récit d'Aétion constitue donc une confirmation de la stratégie choisie par les "Anciens", et la valide comme encore d'actualité à l'époque de Lucien.

\section{Herodotus, une chrie?}

Ce point est intéressant, dans la mesure où il peut nous amener à interpréter différemment l'ensemble de la prolalia et à conjecturer que les développements sur Hérodote et Aétion peuvent aussi être interprétés comme une chrie ( $\left.\chi \rho \varepsilon^{\prime} \alpha\right)$. L'exercice de la chrie, qui suit le récit dans les recueils de progymnasmata postérieurs à Théon, est un exercice plus complexe qui ne consiste pas uniquement à "produire un texte littéraire [...] mais d'en débattre". ${ }^{40}$ Il s'agit d'un rappel ('̊ по paroles ou d'actes, "dont le souvenir mérite de rester dans la mémoire". ${ }^{41}$ Nous aurions, en l'occurrence, une chrie liée aux actes. Voici les éléments de son développement d'après le Pseudo-Hermogène

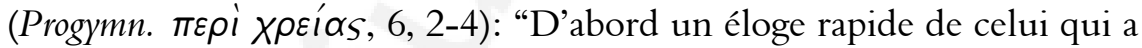
parlé ou agi, ensuite une paraphrase de la chrie elle-même, ensuite la

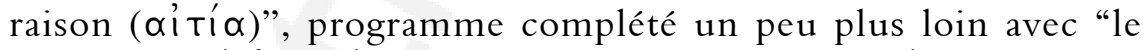

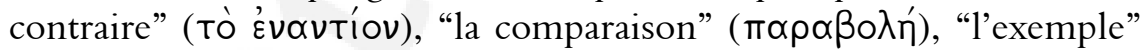
( $\pi \alpha \rho \alpha ́ \delta \varepsilon ı \gamma \mu \alpha)$, puis la précision qu'on "peut encore argumenter avec

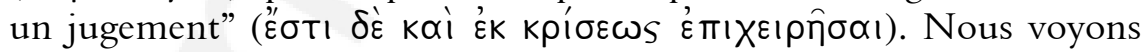
donc, qu'après la paraphrase de la chrie l'orateur avait tout loisir d'élaborer, sous une forme plus ou moins accomplie, un débat, donc une partie délibérative. ${ }^{42}$ Les technographes insistent également sur la

\footnotetext{
${ }^{40}$ Cf. Patillon, op. cit., 2008, p. 221, n. 18.

${ }^{41}$ Cf. Patillon, op. cit., 2008, p. 221, n. 19.

${ }^{42}$ Cf. Patillon, op. cit., 2008, p 72: Par son but, qui est d'exhorter ou de dissuader, le développement appartient au genre délibératif.
} 
portée morale de l'exercice, même si cet aspect n'est pas indispensable (Pseudo-Hermogène, $\pi \varepsilon \rho i$ Xpeías 1,3). ${ }^{43}$ Cela n'est d'ailleurs pas étranger au propos de Lucien: est-il moral de chercher la gloire, et de

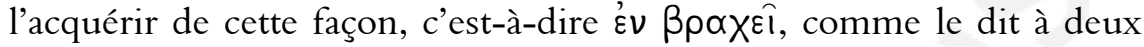
reprises le texte?

Si nous retenons l'hypothèse de la chrie dans ce discours, nous aurions les différentes parties suivantes: l'éloge d'Hérodote qui correspond au prooimion $(\mathbb{S} 1,1.1-8 \mu ı \eta \eta \sigma \alpha i ́ \mu \varepsilon \theta \alpha),{ }^{44}$ la paraphrase de la chrie, autrement dit la narration de l'action "mémorable" d'Hérodote,

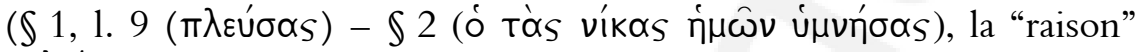
(aitía) en deux temps, $\mathbb{} 2$ ("Tel est le fruit qu'il retira de ses histoires,

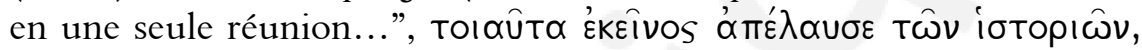

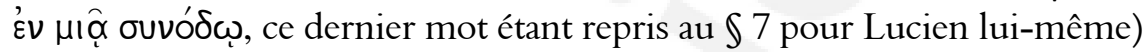
pour le récit concernant Hérodote, puis avec le $\$ 3$ sur les sophistes, qui correspond à l'élargissement normalement attendu pour cette partie; après, commence la partie "délibérative", avec l'objection implicite et la réponse que constitue l'épisode d'Aétion, qui peut être analysé soit comme une comparaison, soit comme un exemple d'acquisition rapide de la célébrité, incluant l'ékphrasis. Cette dernière participe directement à l'argumentation, et en est même une pièce maîtresse, comme Lucien lui-même en avertit tout au long du $₫ 6$, d'abord par sa remarque du

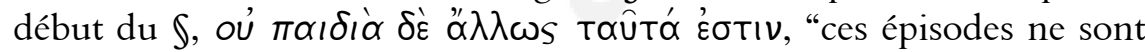
pas une vaine fantaisie du peintre”, ensuite par le commentaire, déjà évoqué, de la fin du paragraphe: le tableau montre Héphaïstos conduisant la mariée Roxane, comme en réalité l'hellanodice Proxénidas conduisit sa fille auprès d'Aétion, et comme Lucien attend que son

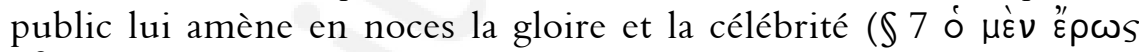
oưtos = Lucien amoureux, désireux de conclure des noces avec les Macédoniens).

Le début du $\int 7$, parfois perçu comme une maladresse, représenterait alors très clairement le signal de la péroraison de la chrie, qui englobe l'ensemble du paragraphe. En effet, le but de la péroraison

\footnotetext{
${ }^{43}$ Cf. Patillon, op. cit., 2008, p. 74: L'exercice de la "chrie" plonge l'élève dans le bain de la vie morale, tant par l'accès à un discours de l'homme surl'homme et sur la vie, que par l'initiation au débat pragmatique.

${ }^{44}$ Cf. Patillon, op. cit., 2008, p. 73: Nous $\gamma$ donnons un exorde, en effet, lorsque nous louons celui qui a parlé ou agi; nous donnons ensuite une narration, lorsque nous paraphrasons la chrie; et nous débattons, même si nous n'abordons pas l'objection, lorsque nous confirmons que nous avons bien parlé ou agi; nous donnons une péroraison, lorsque nous exhortons à mettre en application ce qui a été dit.
} 
est d'exhorter à appliquer ce qui a été dit, à savoir dans le cas de notre

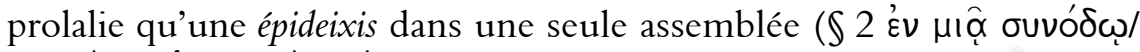

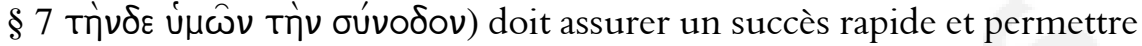
ainsi d'exaucer les vœux de célébrité ( $\varepsilon \dot{u} \times \bar{\eta} s, \$ 1$ et 7 ), et que cela est moral. Son projet a d'autant plus de chances de réussir qu'il s'adresse à un public cultivé ( $(8)$ capable d'apprécier et de goûter à sa juste mesure la prestation et plus particulièrement l'ékphrasis.

Si l'on adopte cette interprétation, il conviendrait par conséquent de revoir le schéma d'ensemble du discours de la façon suivante:

Chrie: $\quad \S 1-7$ qui se décompose de la façon suivante:

I. Prooimion $(\S 1,1.1-8 \mu ı \mu \sigma \alpha i ́ \mu \varepsilon \theta \alpha)$

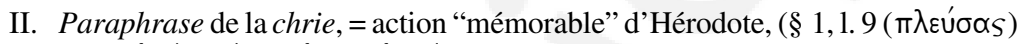

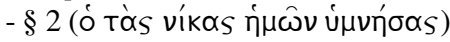

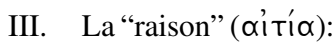

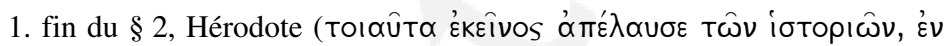

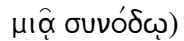

2. $\S 3$, les sophistes, confirmation

IV. Partie "délibérative":

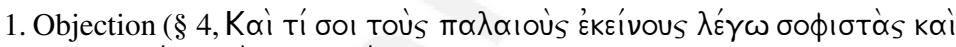

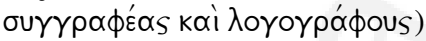

2. Réfutation:

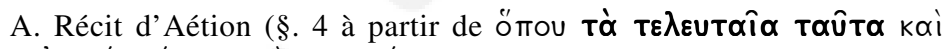

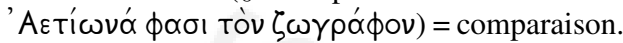

B. Ékphrasis = exemple.

V. Péroraison $(\$ 7)$

\section{Éloge du public: $\S 8$}

Ce discours sous des allures de presque improvisation et de légèreté est remarquable par la complexité de sa construction, l'enchevêtrement des "exercices" qui y est présenté, dont un des éléments, l'ékphrasis, contribue tout autant à la démonstration qu'au plaisir du texte.

Ce qui pourrait confirmer les hypothèses ci-dessus c'est l'examen de Scytha qui, non seulement se trouve également comporter une (petite) ékphrasis, mais qui, peut-être, pourrait se prêter aux mêmes conclusions: le $\int 1$ est un éloge de Toxaris, tel que les chries doivent en comporter, puis suit le récit (annoncé fin du $\ 1=\delta ı \eta \gamma \eta ́ \sigma \alpha \sigma \theta \alpha ı)$ qui, comme dans Herodotus se clôt par l'ékphrasis. Seule une analyse détaillée de ce discours pourrait confirmer cette piste. 


\section{L'enkômion}

La dernière partie des prolaliai est constituée par l'éloge du public, que nous avons déjà évoqué. Peut-on dégager une structure de ces éloges?

Les subdivisions de l'éloge se retrouvent aussi bien dans les progymnasmata que dans les préceptes des théoriciens. ${ }^{45}$ Le schéma suivi par Lucien varie en fonction des discours. Herodotus ( $\mathbb{S} 8)$ commence

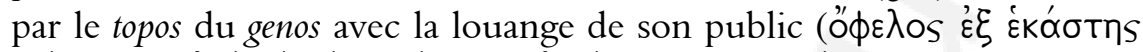

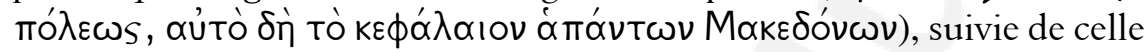

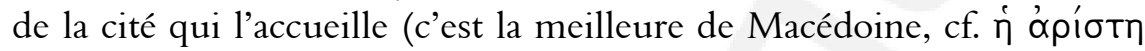

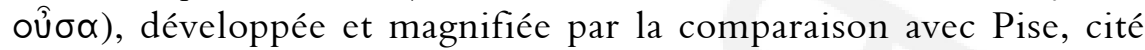
étouffante et rustique. La comparaison entre les cités cède ensuite la place à un parallèle entre les publics, d'un côté les auditeurs macédoniens qui représentent l'élite, de l'autre la populace. Puis vient l'épilogue dans lequel Lucien souhaite bénéficier d'un jugement favorable de la part de son public. L'éloge en est donc réduit essentiellement aux sunkriseis, les comparaisons, car il était impossible dans le cas d'un éloge collectif de faire intervenir l'ensemble des topoi.

Harmonides débute par un exorde à la tonalité très générale $(\mathbb{} 3$, "j'ai regardé quel était dans cette ville le citoyen le plus distingué, celui en qui les autres avaient confiance et qui, à lui seul, suffirait à les remplacer tous. C'est ainsi que je devais très justement trouver en toi la personne qui résume en elle toutes les qualités, c'est-à-dire le

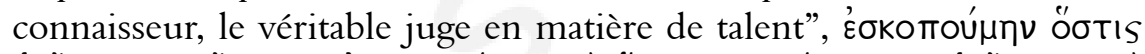

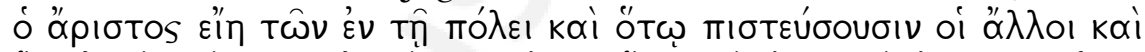

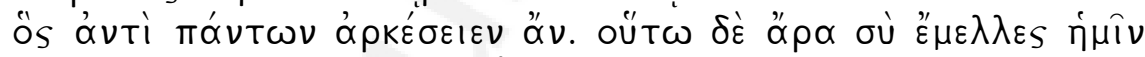

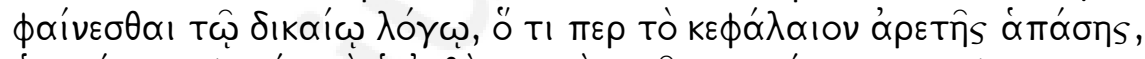

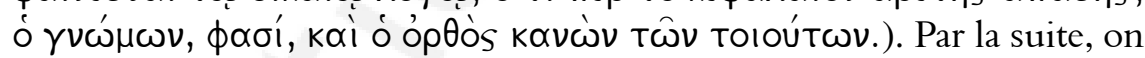
a vu que la paideia du personnage est mise en avant, puis intervient le topos des actions, par la louange des bienfaits du laudandus à l'égard de Samosate (fin du $\ 3$ ), ce qui crée des liens particuliers entre l'orateur et le notable. Enfin, l'épilogue à la manière d'une exhortation $(\mathbb{S} 4$,

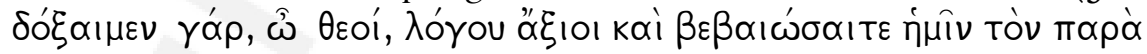

\footnotetext{
${ }^{45}$ Cf. Pernot, op. cit., 1986, p. 35-39. Théon (110, 2-26) présente les lieux de l'éloge dans une répartition tripartite, les biens de l'âme (les sentiments vertueux et les actes qui en sont la suite, la sagesse, la tempérance, le courage, la justice, la piété, la noblesse (eleutherios), la grandeur d'âme (megalophrôn) puis les belles actions.), les biens du corps (la santé, la force, la beauté, la vivacité des sensations.), les biens extérieurs (l'origine, la cité, la nation, le gouvernement, la parenté; puis l'instruction, l'amitié, la gloire, la puissance, la richesse, une mort heureuse).
} 


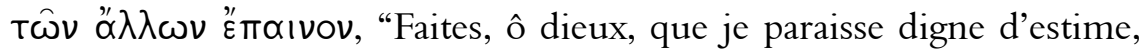
confirmez les éloges que j'ai reçu ailleurs”).

L'enkômion dans Scytha est plus complexe: au $\ 10$ l'éloge, bref,

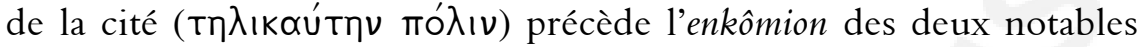
dont Lucien recherche les suffrages, cependant ce n'est pas l'orateur qui va délivrer cet éloge, mais l'ensemble des citoyens qui vont, à l'unisson,

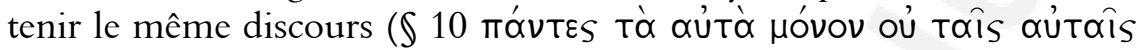

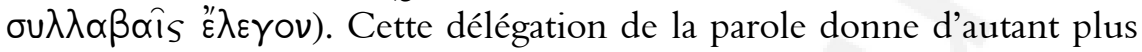
de poids à la louange que Lucien semble n'y avoir aucune part; il ne fait que répéter ce qu'il a entendu à l'envi. Nous avons donc un deuxième éloge (celui des citoyens) à l'intérieur du premier (celui commencé par Lucien) qui se développe de la façon suivante: éloge de la cité, puis des deux personnages avec les topoi de la naissance, de la paideia, leurs actions en faveur de la cité, leur bonté (= âme), leur caractère. Au $\ 11$ l'enkômion se poursuit avec la naissance, puis l'éloge du père et la mise en valeur de sa sagesse, ensuite celui du fils avec l'insistance sur sa

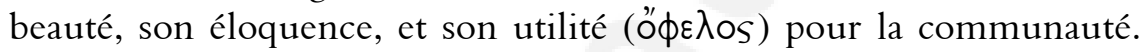
Suit une rapide conclusion assurant Lucien du succès s'il s'appuie sur ces deux personnalités. L'enkômion premier, celui de Lucien, reprend à la fin du paragraphe pour, cette fois, parler en connaissance de cause (“j'en ai fait l'expérience moi-même”) et attester de la véracité de tout ce qu'il a entendu. Un éloge, donc, qui semble parfois proche de la flatterie, tant il peut apparaître excessif, ce qui explique que, très habilement, Lucien ne le prononce pas lui-même mais en délègue les propos aux citoyens.

Dans les trois prolaliai Lucien met en place une partie encomiastique fort différente l'une de l'autre, ce qui souligne qu'il ne se sent pas tenu par un enkômion académique, même s'il ne méconnaît pas le genre, loin de là, comme l'atteste Scytha. Cela tient à la forme même de la prolalia dont on a eu l'occasion de souligner la souplesse et le peu de contraintes qui s'y appliquent. La partie encomiastique en est certainement un des exemples les plus manifestes.

L'éloge que l'on trouve dans le Scythe est peut-être le plus riche d'enseignements dans la mesure où l'on peut y distinguer avec netteté les strates de l'éloge tel qu'il était enseigné dans les écoles de rhétorique, en même temps qu'on ne peut que constater la totale liberté de l'orateur. Non seulement Lucien y adopte cette curieuse structure qui fait que l'éloge 2, fait par les concitoyens des deux laudandi, est enchâssé dans celui qu'il est en train de faire, mais, de plus, les parties, tout en étant pour la plupart répertoriées dans les manuels de progymnasmata ou par Ménandros II, se déploient de façon singulière, puisqu'elles sont alternativement adressées 
au père puis au fils. Il est curieux de noter, d'ailleurs, que, contrairement à ce qu'on pourrait attendre, l'éloge du fils est plus appuyé que celui du père, alors qu'on aurait pu penser que ce dernier avait davantage de poids dans la cité. Certainement n'en était-il rien.

\section{Conclusion}

Quelques remarques s'imposent à la suite de cette enquête.

Le schéma d'ensemble des prolaliai étudiées - mais au-delà de l'ensemble des prolaliai de Lucien -, est globalement semblable, puisque leur objectif est de s'attirer la bienveillance d'un public et de souhaiter le succès pour la lecture ou la récitation qui doit suivre. En tant que sophiste, Lucien n'ignore aucune des règles d'un discours qui précisément se distingue par sa grande liberté de ton et d'allure. En ce sens, on peut dire que Lucien se "fabrique" en quelque sorte son schéma de prolalia, canevas qu'il est prêt à appliquer en toutes circonstances, sa recette en somme. Cela explique les nombreux points communs que l'on peut observer entre ces discours, sur lesquels il n'est pas utile de revenir. Mais cela n'est pas propre aux prolaliai ni à Lucien. Les sophistes de la Seconde Sophistique ont tous la même formation, ils ont étudié à partir des mêmes manuels de progymnasmata, ils apprennent tous les même exercices et sont donc amenés à traiter de façon plus ou moins semblable, dans les grandes lignes, leurs discours, en fonction de la caractéristique de ces derniers; il est évident qu'on ne composera pas un éloge funèbre comme une déclamation. En même temps, le bon orateur est celui qui sait utiliser ces contraintes, qui peuvent parfois sembler un carcan étroit, pour manifester son talent, et adapter son discours au public, aux circonstances, à son projet, bref, à tout ce qui fait pour la rhétorique grecque le kairos.

On peut appliquer ces constatations à propos des prolaliai de Lucien. Les nombreux points communs entre les trois discours ne peuvent masquer l'individualisation qui les caractérise, et qui doit, d'ailleurs, relativiser l'emploi des prolaliai comme prooimia pour différents discours. Nous avons pu constater qu'aussi bien dans le vocabulaire utilisé, que dans la manière d'équilibrer les différentes parties dans les discours, ou encore dans le traitement de l'éloge, ou l'inclusion ou non d'une ékphrasis, Lucien fait véritablement œuvre originale de composition et ne se contente pas d'ajouter un topos à un autre. Les circonstances, que nous avons tenté de mettre en évidence, commandent toujours l'écriture. 
Certainement ces discours n'ont pas encore livré tous leurs ressorts quant à la mise en œuvre rhétorique, l'analyse d'Herodotus montre combien, sous leur apparente simplicité - celle de la prolalia -, ils recèlent d'originalité et, surtout, de complexité. Nous avons touché cette complexité du doigt à travers l'étude de la structure d'Herodotus et la conjecture qui en découle - voir dans ce discours non pas un récit suivi d'une ékphrasis, interprétation traditionnelle, mais bien plutôt une chrie, exercice plus sophistiqué -, indique combien Lucien sait cacher un art consommé de la rhétorique derrière une apparente légèreté. C'est cette capacité à brouiller les pistes qui lui a procuré une part de notoriété: les spectateurs attendaient de lui des nouveautés, des morceaux originaux et pétillants (cf. Zeuxis 1), ce qu'en apparence il fait dans ces prolaliai, et la captatio benevolentiae qui s'adresse à un public de gens cultivés, nourris de la paideia, est plus qu'un jeu d'enfant, un simple paidia, comme il nous en avertit lui-même dans Herodotus 6.

\section{Bibliographie}

ANDERSON, G. Patterns in Lucian's "Prolaliae”. Philologus, Berlin, vol. 121, p. 313315, 1977.

BRANHAM, R. B. Introducing a Sophist: Lucian's prologues. Transactions of the American Philological Association, Cleveland (Ohio), vol. 115, p. 237-243, 1985.

BOMPAIRE, J. Lucien écrivain: imitation et création. Paris: E. de Boccard, 1958.

BOULANGER, A. Aelius Aristide et la seconde sophistique dans la province d'Asie au II' s. de notre ère. Paris: E. de Boccard, 1923.

CHAMBRY, E. Lucien de Samosate: Cuvres complètes. III volumes. Paris: Garnier, s.d. DESBORDES, F. La rhétorique antique: l'art de persuader. Paris: Hachette, 1996.

JONES, C. P. Culture and society in Lucian. Cambridge Mass.: Harvard University Press, 1986.

MRAS, K. Die "prolaliai" bei den griechischen Schriftstellern. Wiener Studien LXIV. Vienne: Verlag der Österreichischen Akademie der Wissenschaften, 1949, p. 71-81.

NESSELRATH, H. G. Lucian's introductions. In: RUSSELL, D. A. (org.). Antonine Literature. Oxford: Clarendon Press, 1990, p. 110-140.

PATILLON, M.; BOLOGNESI, G. Aelius Théon, Progymnasmata. Paris: CUF, 1997. PATILLON, M. Hermogène, L'art rhétorique. Traduction française intégrale. LausanneParis: L'âge d'homme, 1997.

PATILLON, M. Corpus Rhetoricum. Anonyme Préambule à la rhétorique. Aphthonios Progymnasmata. Pseudo-Hermogène Progymnasmata. Paris: CUF, 2008. 
PERNOT, L. La rhétorique de l'éloge dans le monde gréco-romain. 2 tomes. Paris: Institut d'Études augustiniennes, 1993.

PERNOT, L. L'éloge chez Ménandre le rhéteur. REG, Paris, vol. 99, p. 33-53, 1986. RABE, H. Hermogenis opera. Leipzig: Teubner, 1913.

REARDON, B. P. Courants littéraires grecs des II et III s. ap. J.-C. Paris: Les Belles Lettres, 1971.

ROTHSTEIN, M. Quaestiones Lucianeae. Berlin: Mayer et Muller, 1888 [Prolaliai p. 116-123].

RUSSELL, D. A.; WILSON, N. G. Menander Rhetor. Edited with translation and commentary. Oxford: Clarendon Press, 1981.

SCHISSEL, O. Novellenkränze Lukians. Rhetorische Forschung. Halle: M. Niemeyer, 1912.

SCHMID, W. Bemerkungen über Lucians Leben und Schriften. Philologus, Berlin, vol. 50, p. 297-319, 1891.

SCHWARTZ, J. Biographie de Lucien de Samosate. Latomus, 83. Bruxelles: Berchem, 1965.

SPENGEL, L. Rhetores graeci. Leipzig: Teubner, 1853-1856 (repr. Francfort-sur-le Main, 1966, p. 57-82).

STOCK, A. De Prolaliarum usu rhetorico. Königsberg: ex officina Hartungiana, 1911.

VIX, J.-L. L'enseignement de la rhétorique au II' siècle ap. J.-C. à travers les discours 30-33 d'Aelius Aristide. Recherches sur les Rhétoriques religieuses, 13. Turnhout: Brepols, 2010.

WALZ, C. Rhetores Graeci. 9 vol. Stuttgart/ Tübingen: J.G. Cotta, 1832-1836 (repr. Osnabrück, 1968, vol. VIII, p. 520-577). 